\title{
Analysis of magnetohydrodynamic natural convection in closed cavities through integral transforms
}

\author{
Carlos Frederico Trotta Matt ${ }^{\mathrm{a}}$, João Nazareno Nonato Quaresma ${ }^{\mathrm{b}}$, Renato Machado Cotta ${ }^{\mathrm{c}, \mathrm{d}, *}$ \\ ${ }^{a}$ Electric Power Research Center (CEPEL), Department of Lines and Substations, 21941-590 Rio de Janeiro, RJ, Brazil \\ ${ }^{\mathrm{b}}$ Universidade Federal do Pará, School of Chemical Engineering (FEQ/UFPA), 66075-110 Belém, PA, Brazil \\ ${ }^{\mathrm{C}}$ Universidade Federal do Rio de Janeiro, POLI \&' COPPE/UFRJ, Department of Mechanical Engineering, 21945-970 Rio de Janeiro, RJ, Brazil \\ ${ }^{\mathrm{d}}$ Universidade Federal do Rio de Janeiro, Interdisciplinary Nucleus for Social Development, NIDES/CT, Rio de Janeiro, RJ, Brazil
}

\section{A R T I C L E I N F O}

\section{Article history}

Received 2 March 2017

Received in revised form 10 May 2017

Accepted 11 May 2017

Available online 3 June 2017

\section{Keywords}

Magnetohydrodynamics

Natural convection

Closed cavities

Electrically-conducting fluid

Hybrid methods

Integral transforms

\begin{abstract}
A B S T R A C T
A hybrid numerical-analytical solution is proposed to analyze MHD (magnetohydrodynamic) natural convection of an electrically-conducting fluid within a square cavity, differentially heated at the sidewalls and subjected to an inclined external magnetic field. The first goal is to expand the spectrum of application of the so called Generalized Integral Transform Technique (GITT), dealing with a multiphysics formulation, while further demonstrating the relative merits of the proposed eigenfunction expansion approach in handling highly nonlinear and coupled systems of partial differential equations. The second goal is to provide a set of benchmark results in this important application for quantities of practical interest in determining the heat transfer rates, such as the average Nusselt number. The two-dimensional steady state equations are written in dimensionless form using the streamfunction-only formulation and are subsequently solved with the GITT approach, under automatic relative error control. Critical comparisons are performed against previous work reported in the literature, both computational and experimental, together with the corresponding physical interpretations, for different values of the governing parameters, such as Grashof number, Hartmann number, Prandtl number, and magnetic field inclination angle.
\end{abstract}

(C) 2017 Elsevier Ltd. All rights reserved.

\section{Introduction}

Magnetohydrodynamics (MHD) deals with the motion of electrically-conducting fluids under the influence of externally applied electromagnetic fields. Examples of such fluids include ionized gases (plasma), liquid metals, saline water, and electrolytes. A quite comprehensive review on MHD may be found in the monograph by Davidson [1]. MHD is currently viewed as a particular case of a more general continuum mechanics-based theoretical framework referred to as Unified Electro-Magneto-Fluid Dynamics (EMFD) [2-5]. MHD natural convection inside closed cavities has received considerable attention in the past few decades because it occurs in numerous engineering applications such as in the liquid metal cooling of nuclear reactors and electric equipment [6-10], the manufacturing process of high-quality crystals [9,11-12], and magnetic-levitation casting [13], to name just a few.

The literature on MHD natural convection inside closed cavities is quite extensive and a detailed review is beyond the scope of the

\footnotetext{
* Corresponding author at: Universidade Federal do Rio de Janeiro, POLI \& COPPE UFRJ, Department of Mechanical Engineering, 21945-970 Rio de Janeiro, RJ, Brazil.

E-mail address: cotta@mecanica.coppe.ufrj.br (R.M. Cotta).
}

current work. The vast majority of previous studies focuses on twodimensional laminar and incompressible flow of electricallyconducting fluids inside cavities, differentially heated either from the sidewalls or from its top and bottom walls, and subjected to either transverse, parallel or inclined magnetic fields with respect to the gravitational acceleration vector. Oreper and Szekely [12] were the first to numerically investigate the effect of an externally imposed magnetic field (transversal to gravity) on the natural convection inside a square cavity differentially heated from the sidewalls. Ozoe and Okada [11] investigated the MHD natural convection in three-dimensional cubic enclosures differentially heated from two vertical walls and under magnetic fields oriented along the principal axis of the cubic enclosure. Alchaar et al. [9] investigated the MHD natural convection inside a shallow cavity heated from below and cooled from the top, and subjected to an inclined magnetic field. Al-Najem et al. [10] investigated the MHD natural convection within a tilted square cavity differentially heated from its vertical walls and permeated by an inclined external magnetic field. Colaço et al. [14] revisited the MHD natural convection problem investigated in [10] and solved the MHD governing equations using a meshless method with radial basis functions (RBF) [15]. Results for the velocity and temperature 


\begin{tabular}{|c|c|}
\hline \multicolumn{2}{|c|}{ Nomenclature } \\
\hline$A_{i j}$ & integral coefficient given by Eq. (42a) \\
\hline $\mathbf{B}_{0}^{*}, \mathbf{B}^{*}, \mathbf{B}^{\prime *}$ & induced magnetic fields \\
\hline$B_{i j}$ & integral coefficient given by Eq. (42b) \\
\hline $\mathrm{C}_{\mathrm{ijk}}$ & integral coefficient given by Eq. (42c) \\
\hline$D_{i j k}$ & integral coefficient given by Eq. (42d) \\
\hline $\begin{array}{l}\mathbf{e}_{x_{*}}, \mathbf{e}_{y *} \\
\mathbf{E}^{*}\end{array}$ & $\begin{array}{l}\text { unit vectors along the } x^{*} \text { and } y^{*} \text { axes } \\
\text { electric field strength }\end{array}$ \\
\hline$E_{i j k}$ & integral coefficient given by Eq. (42e) \\
\hline$\overline{\mathrm{f}}_{i}$ & transformed boundary condition given by Eq. (42i) \\
\hline $\mathrm{F}_{\mathrm{ij}}$ & integral coefficient given by Eq. (42f) \\
\hline$g$ & gravity acceleration \\
\hline $\mathrm{G}_{\mathrm{ijk}}$ & integral coefficient given by Eq. (42g) \\
\hline $\mathrm{Gr}$ & Grashof number \\
\hline $\mathbf{H}_{0}^{*}$ & magnetic field strength \\
\hline На & Hartmann number \\
\hline $\mathrm{H}_{\mathrm{ijk}}$ & integral coefficient given by Eq. (42h) \\
\hline $\mathrm{I}_{\mathrm{ij}}$ & integral coefficient given by Eq. (44c) \\
\hline $\mathbf{J}^{*}$ & electric current density \\
\hline$L$ & cavity length \\
\hline $\mathrm{Nu}, \overline{N u}_{\mathrm{x}=0}$ & local and average Nusselt numbers, respectively \\
\hline$N_{\Phi i}, N_{\Gamma i}$ & $\begin{array}{l}\text { normalization integrals for eigenfunctions } \Phi_{i}(y) \text { and } \\
\Gamma_{\mathrm{i}}(y) \text {, respectively }\end{array}$ \\
\hline$N_{\psi}, N_{\mathrm{T}}$ & $\begin{array}{l}\text { truncation orders for the streamfunction and tempera- } \\
\text { ture fields, respectively }\end{array}$ \\
\hline$p^{*}\left(x^{*}, y^{*}\right)$ & pressure field \\
\hline $\mathrm{Pr}$ & Prandtl number \\
\hline $\mathrm{Ra}=\mathrm{GrPr}$ & Rayleigh number \\
\hline $\operatorname{Re}$ & Reynolds number \\
\hline$R e_{m}$ & magnetic Reynolds number \\
\hline$T^{*}\left(x^{*}, y^{*}\right)$ & temperature field \\
\hline$T_{\text {ref }}^{*}$ & reference temperature \\
\hline
\end{tabular}

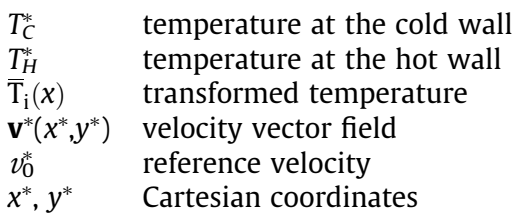

\section{Greek letters}

$\alpha \quad$ fluid thermal diffusivity

$\alpha_{i} \quad$ eigenvalues for the streamfunction expansion

$\beta \quad$ coefficient of thermal expansion of fluid

$\beta_{\mathrm{i}} \quad$ eigenvalues for the temperature expansion

$\gamma \quad$ magnetic field inclination angle with respect to $x$-axis

$\sigma_{e} \quad$ fluid electric conductivity

$\mu_{e} \quad$ fluid magnetic permeability

$v \quad$ fluid kinematic viscosity

$v_{m} \quad$ fluid magnetic diffusivity

$\rho \quad$ fluid density

$\Phi_{i}(y) \quad$ eigenfunction for the streamfunction expansion

$\Gamma_{i}(y) \quad$ eigenfunction for the temperature expansion

$\bar{\Psi}_{\mathrm{i}}(\mathrm{x}) \quad$ transformed streamfunction

$\psi^{*}\left(x^{*}, y^{*}\right)$ streamfunction field
Subscripts and superscripts
$\mathrm{i}, \mathrm{j}, \mathrm{k} \quad$ orders from eigenvalue problems
ref quantity evaluated at reference temperature $T_{\text {ref }}{ }^{*}$
$\sim \quad$ normalized eigenfunctions
* dimensional quantities
- $\quad$ transformed quantities

distributions as well as for the average Nusselt number at the solid walls of the cavity have been reported in the literature, offering reference values for comparison and verification tasks. The effects of the governing parameters, namely, the Grashof number, the Hartmann number, the inclination angle of either the cavity or the magnetic field on the convective heat transfer rate, represented by the average Nusselt number, are well documented. The reported results mostly indicate that an external magnetic field, independent of its orientation, contributes to reducing the convective heat transfer through the cavity. The extent of heat transfer reduction depends strongly upon the imposed magnetic field strength. Magnetic fields oriented perpendicular to the heat flow direction are the most effective in suppressing convective heat transfer. For cavities heated from the bottom and cooled from the top, the results reported in [9] also indicate that the convection modes within the cavity depend strongly upon both the strength and inclination of the magnetic field.

From the literature review on MHD natural convection within closed cavities, the following remarks should be summarized. Firstly, the vast majority of the previous works relies on either finite-difference or finite-volume schemes to solve the governing equations. Such classical numerical schemes require spatial discretization of the domain and an approach to handle the velocity-pressure coupling. A few authors avoided the velocitypressure coupling by rewriting the governing equations using the streamfunction-vorticity formulation. However, the boundary conditions adopted for the vorticity field at the solid walls are rarely reported, with a noteworthy exception in [11]. Secondly, few works have attempted to solve the governing equations using the streamfunction-only formulation [14], which has the advantage of not requiring boundary conditions for the vorticity field at the solid walls, albeit it requires a special scheme to accurately approximate fourth-order derivatives [14]. Thirdly, there are nonnegligible discrepancies amongst the numerical results reported in the literature for the average Nusselt number, with relative deviations ranging from $2.9 \%$ to $32 \%$ [14]. Fourth, the majority of previous works reports numerical results only for the special case in which the magnetic field induced by fluid flow is negligible compared to the imposed one (inductionless approximation), decoupling Maxwell's equations from the Navier-Stokes equations for fluid flow. The current work comprises a detailed derivation of the conditions for this assumption to be valid.

Despite the extensive progress achieved by discrete numerical methods, analytical-type approaches for diffusion and convection-diffusion problems have been progressively advanced and extended, in part motivated by offering benchmark results for verification and calibration of the more flexible numerical methods. Powerful hybrid analytical-numerical schemes have emerged from the combination of classical analytical methods with modern computational methods for ordinary differential equations, benefiting as well from modern symbolic computation platforms. The Generalized Integral Transform Technique (GITT) is one such a hybrid method for solving linear or nonlinear diffusion and convection-diffusion problems, which has been developed for the last three decades, dealing with various classes of problems in heat and fluid flow, as reviewed in different sources [16-24]. A few contributions are here briefly mentioned, which have a closer connection to the problem under consideration. Natural convection inside cavities was first dealt with the GITT in [25], for a two-dimensional rectangular porous region with internal heat generation. Transient analysis of natural convection in porous cavities was then analyzed through the hybrid approach, both for 
two-dimensional [26] and three-dimensional [27] geometries. The streamfunction only formulation was preferred in the twodimensional situations $[25,26]$ and the vorticity-vector potential formulation was adopted for the three-dimensional problem [27]. Leal et al. [28] examined the convergence characteristics of the GITT solution for the steady laminar natural convection of a Newtonian fluid inside rectangular void enclosures differentially heated at the vertical walls. Further, Leal et al. [29] have extended the GITT approach to transient laminar natural convection with variable physical properties inside two-dimensional cavities. Recently, An et al. [30] revisited the natural convection problem investigated in Refs. [28,29], but including volumetric heat generation inside differentially heated and isothermal cavity walls. Such contributions illustrate the accuracy and robustness of the GITT approach in dealing with natural convection problems. In addition, Lima and Rêgo [31] have applied the GITT approach to investigate the MHD flow and heat transfer in the entrance region of a channel. Also closely related to the eigenfunction expansion basis here adopted, though not directly related to natural convection, the GITT approach has been successfully employed in handling structural dynamics problems, such as the dynamic transient response of axially moving beams [32,33], axially moving orthotropic plates [34], damaged Euler-Bernoulli beams [35], cantilever beams with eccentric tip mass [36], general one-dimensional slender structures with inertia, damping and stiffness elements at the boundaries [37], fluid-conveying pipes [38], and overhead transmission lines [39].

The GITT approach is rooted in the Classical Integral Transform Technique (CITT) for linear diffusion problems, extensively discussed in the treatise by Mikhailov and Özisik [40]. It was developed to overcome formulation complexities that were before supposed to be solvable solely by discrete-type methods, including nonlinear physical properties, moving boundaries, irregular geometries and nonlinear convective terms [16-24]. The main idea in the GITT is to eliminate all but one independent variable with an appropriate choice of auxiliary eigenvalue problem and definition of an inverse-transform pair. The auxiliary eigenvalue problem provides the orthogonal eigenfunctions for the series expansions of the unknown potentials. After integral transformation, the original system of partial differential equations (PDEs) is transformed into a system of coupled ordinary differential equations (ODEs). With the inversion formula, a priori available, the dependence of the unknown potentials on the eliminated independent variables may be recovered in analytical form. Hence, the numerical task comprises only the solution of a system of ordinary differential equations, which nowadays may be readily accomplished with different highly accurate and robust ODE solvers. The numerical evaluation of coefficients appearing in the resulting system of ODEs may deteriorate computational efficiency for nonlinear formulations. However, accurate and computationally efficient implementations can be readily achieved through symbolic manipulation platforms and semi-analytical integration schemes. The relative merits of the GITT approach over purely numerical schemes comprises avoiding 2-D or 3-D spatial discretization, yielding automatic global accuracy control, and offering mild increase in computational cost with increasing number of independent variables.

Here, MHD natural convection of an electrically-conducting fluid within a square cavity is revisited [41], for heated and cooled sidewalls, and subjected to an inclined external magnetic field. The governing equations are written in dimensionless form using the streamfunction-only formulation and subsequently solved with the GITT approach. The first main goal is to expand the field of application of the GITT approach to a complex multiphysics problem, and to further demonstrate the relative merits of the proposed eigenfunction expansion approach in handling highly nonlinear
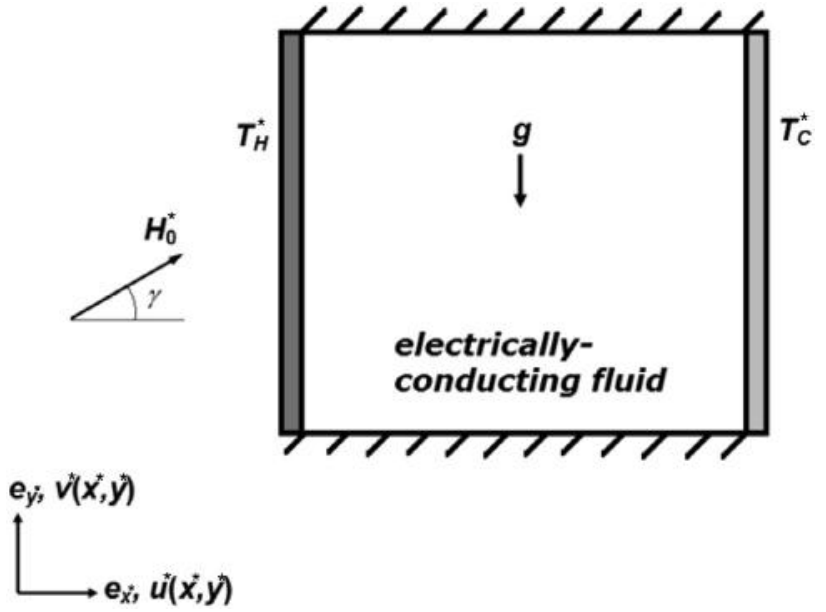

Fig. 1. MHD natural convection inside a differentially heated square cavity filled with an electrically-conducting fluid.

and coupled systems of partial differential equations. Another equally important goal is to provide new benchmark results for relevant quantities such as heat transfer rates, represented by the average Nusselt number, with the corresponding physical behavior interpretation.

The current work is organized as follows. Section 2 presents the mathematical formulation of the physical problem. Section 3 describes the hybrid solution of the governing equations formulated in Section 2 through the GITT approach. In Section 4, the numerical results for velocity, temperature and average Nusselt numbers are reported, discussed, and critically compared against previous ones reported in the literature. Section 5 summarizes the main conclusions and provides suggestions for future research work.

\section{Mathematical formulation}

The physical problem here addressed stands for laminar, steady and incompressible flow of an electrically-conducting fluid within a differentially heated square cavity. The square cavity has length $L$ in the $x^{*}$ - and $y^{*}$-directions, and is of infinite extent in the $z^{*}$-direction, as sketched in Fig. 1. The top and bottom walls of the cavity are assumed to be thermally insulated, whereas the left and right vertical walls are maintained at the prescribed uniform temperatures $T_{H}^{*}$ (hot wall) and $T_{C}^{*}$ (cold wall), respectively. It is also assumed that the cavity walls are electrically insulated. The entire system (fluid and solid walls) is subjected to an external magnetic field of uniform strengths $H_{0 x}^{*}$ and $H_{0 y}^{*}$ acting in the $x^{*}$ - and $y^{*}$-directions, respectively. Let us denote by $\mathbf{B}_{0}^{*}$ the induced magnetic field due to the externally applied magnetic field $\mathbf{H}^{*}=H_{0 x}^{*} \mathbf{e}_{x *}+H_{0 x}^{*} \mathbf{e}_{y_{*}}$, where $\mathbf{e}_{x *}$ and $\mathbf{e}_{y *}$ denote the unit vectors along the $x^{*}$ - and $y^{*}$-directions. Neglecting fluid magnetization, then $\mathbf{B}_{0}^{*}=\mu_{e} \mathbf{H}_{0}^{*}$, where $\mu_{e}$ stands for the magnetic permeability of the fluid.

When an electrically-conducting fluid moves with velocity $\mathbf{v}^{*}$ through an imposed magnetic field, its positive and negative charges are each accelerated such that their average motion gives rise to an electric current density $\mathbf{J}^{*}$ given by Ohm's law as $\mathbf{J}^{*}=\sigma_{e}\left(\mathbf{E}^{*}+\mathbf{v}^{*} \times \mathbf{B}^{*}\right)$. The induced magnetic field $\mathbf{B}^{*}$ may be written as $\mathbf{B}^{*}=\mathbf{B}_{0}^{*}+\mathbf{B}^{\prime *}$. The symbol $\sigma_{e}$ denotes the electric conductivity of the fluid and the vector quantity $\mathbf{B}^{\prime *}$ is the induced magnetic field due to the electric current $\mathbf{J}^{*}$ flowing through the fluid. ${ }^{1}$.

\footnotetext{
1 The ideal MHD flow is a particular case for which one assumes that the fluid behaves as a perfect electrical conductor (i.e., $\sigma_{e} \rightarrow \infty$ ) such that in order to $\mathbf{J}^{*}$ remains finite, Ohm's law leads to $\mathbf{E}^{*}+\mathbf{v}^{*} \times \mathbf{B}^{*}=\mathbf{0}$.
} 
The following assumptions are made: (i) the fluid is Newtonian; (ii) fluid properties are constant and evaluated at the reference temperature $T_{\text {ref }}^{*}$; the density dependence on temperature that promotes the effect of buoyancy forces is treated according to Boussinesq's approximation, i.e., $\rho\left(T^{*}\right) \approx \rho_{\text {ref }}+\left.\left(\partial \rho / \partial T^{*}\right)\right|_{T^{*}}=T_{r e f}^{*}\left(T^{*}-T_{r e f}^{*}\right)$ and $\rho_{\text {ref }}=\rho\left(T_{r e f}^{*}\right)$; (iii) viscous dissipation and Joule heating are neglected. An assumption commonly adopted in the literature and referred to as the inductionless approximation states that the induced magnetic field $\mathbf{B}^{* *}$ is negligible in comparison with $\mathbf{B}_{0}^{*}$, i.e., $\mathbf{B}^{*} \approx \mathbf{B}_{0}^{*}$. However, the more general formulation without the inductionless approximation will be here retained along the derivations.

The governing equations to be solved are the conservation of mass, momentum, and energy for the fluid flow, and the conservation of electric charges, Ohm's law and Ampère-Maxwell's equations in a moving medium for the electromagnetic field, which may be written in (dimensional) vector form as follows [12,14]:

$\nabla^{*} \cdot \mathbf{v}^{*}=0$

$\mathbf{v}^{*} \cdot \nabla^{*} \mathbf{v}^{*}=-\frac{1}{\rho_{\text {ref }}} \nabla^{*} p^{*}+v \nabla^{2 *} \mathbf{v}^{*}+g \beta\left(T^{*}-T_{r e f}^{*}\right) \mathbf{e}_{y^{*}}+\frac{1}{\rho_{\text {ref }}} \mathbf{J}^{*} \times \mathbf{B}^{*}$

$\mathbf{v}^{*} \cdot \nabla^{*} T^{*}=\alpha \nabla^{2 *} T^{*}$

$\nabla^{*} \cdot \mathbf{J}^{*}=0$

$\mathbf{J}^{*}=\sigma_{e}\left(\mathbf{E}^{*}+\mathbf{v}^{*} \times \mathbf{B}^{*}\right)$

$\nabla^{*} \times \mathbf{B}^{*}=\mu_{e} \mathbf{J}^{*}$

The symbols $\rho_{\text {ref, }}, v \equiv \mu / \rho_{\text {ref }}, \quad \beta \equiv-\rho^{-1}\left(\partial \rho / \partial T^{*}\right) \mid T^{*}=T_{\text {ref }}^{*}$ and $\alpha$ denote, respectively, the density, the kinematic viscosity, the volumetric coefficient of thermal expansion and the thermal diffusivity of the conducting fluid (all evaluated at the reference temperature $T_{r e f}^{*}$ ); and $p^{*}$ and $T^{*}$ denote the pressure and temperature fields. The last two terms appearing in Eq. (2) account for the body forces due to gravity and the induced magnetic field (Lorentz body force). There is also one more equation to be satisfied, namely, the Faraday's law that relates the electric field $\mathbf{E}^{*}$ to the induced magnetic field $\mathbf{B}^{*}$

$\frac{\partial \mathbf{B}^{*}}{\partial t}+\nabla^{*} \times \mathbf{E}^{*}=0$

Faraday's law imposes that the induced magnetic field is divergence-free, i.e., $\nabla^{*} \cdot \mathbf{B}^{*}=0$, which is a mathematical expression for the conservation of magnetic flux. Combining Eqs. (5) and (6) one may rewrite Eq. (7) as

$\frac{\partial \mathbf{B}^{*}}{\partial t}+\frac{1}{\mu_{e} \sigma_{e}} \nabla^{*} \times\left(\nabla^{*} \times \mathbf{B}^{*}\right)-\nabla^{*} \times\left(\mathbf{v}^{*} \times \mathbf{B}^{*}\right)=0$

By using the vector calculus identity $\nabla^{*} \times\left(\nabla^{*} \times \mathbf{B}^{*}\right)=\nabla^{*}\left(\nabla^{*} \cdot \mathbf{B}^{*}\right)-$ $\nabla^{2 *} \mathbf{B}^{*}$ and the divergence-free constraint for the induced magnetic field $\mathbf{B}^{*}$, one may finally rewrite Faraday's law as:

$\frac{\partial \mathbf{B}^{*}}{\partial t}=\frac{1}{\mu_{e} \sigma_{e}} \nabla^{2 *} \mathbf{B}^{*}+\nabla^{*} \times\left(\mathbf{v}^{*} \times \mathbf{B}^{*}\right)$

Notice that the governing partial differential equation for the induced magnetic field comprises both diffusive and convective terms and it is coupled to the momentum conservation equations through the convective term $\nabla^{*} \times\left(\mathbf{v}^{*} \times \mathbf{B}^{*}\right)$. For an externally applied time-independent magnetic field, one may further simplify Eq. (9) to:

$\nabla^{2 *} \mathbf{B}^{*}+\mu_{e} \sigma_{e} \nabla^{*} \times\left(\mathbf{v}^{*} \times \mathbf{B}^{*}\right)=0$
By combining Eqs. (4) and (5), one obtains

$\nabla^{*} \cdot \mathbf{E}^{*}+\nabla^{*} \cdot\left(\mathbf{v}^{*} \times \mathbf{B}^{*}\right)=0$

For a two-dimensional MHD flow, it is easy to verify that $\nabla^{*} \cdot\left(\mathbf{v}^{*} \times \mathbf{B}^{*}\right)=0$; hence, the conservation of electric charges reduces to $\nabla^{*} \cdot \mathbf{E}^{*}=0$.

Faraday's law for time-independent magnetic fields, $\nabla^{*} \times \mathbf{E}^{*}=0$, implies that $\mathbf{E}^{*}=-\nabla^{*} \phi_{\mathrm{E}}$ where $\phi_{\mathrm{E}}$ denotes the electric potential; thence, the conservation of electric charges, $\nabla^{*} \cdot \mathbf{E}^{*}=0$, reduces to the Laplace equation $\nabla^{2 *} \phi_{\mathrm{E}}=0$. For electrically-insulating boundaries, on which the derivative of the electric potential along the outward normal $\mathbf{n}$ is identically zero (i.e., $\partial \phi_{\mathrm{E}} / \partial \mathbf{n}=\nabla^{*} \phi_{\mathrm{E}} \cdot \mathbf{n}=0$ ), the unique solution to $\nabla^{2 *} \phi_{\mathrm{E}}=0$ is simply $\nabla^{*} \phi_{\mathrm{E}}=0$. It follows thus that the electric field $\mathbf{E}^{*}$ vanishes everywhere. Finally, by further inserting Eq. (5) with $\mathbf{E}^{*}=0$ into Eq. (2), one arrives at the modified Navier-Stokes equations for natural convection flows of electrically-conducting fluids subjected to a magnetic field

$\mathbf{v}^{*} \cdot \nabla^{*} \mathbf{v}^{*}=-\frac{1}{\rho_{\text {ref }}} \nabla^{*} p^{*}+v \nabla^{2 *} \mathbf{v}^{*}+g \beta\left(T^{*}-T_{r e f}^{*}\right) \mathbf{e}_{y^{*}}+\frac{\sigma_{e}}{\rho_{\text {ref }}}\left(\mathbf{v}^{*} \times \mathbf{B}^{*}\right) \times \mathbf{B}^{*}$

Thus, the system of partial differential equations to be solved for a two-dimensional MHD flow, in the presence of an externally applied time-independent magnetic field, may be written as follows:

$\nabla^{*} \cdot \mathbf{v}^{*}=0$

$\mathbf{v}^{*} \cdot \nabla^{*} \mathbf{v}^{*}=-\frac{1}{\rho_{\text {ref }}} \nabla^{*} p^{*}+v \nabla^{2 *} \mathbf{v}^{*}+g \beta\left(T^{*}-T_{r e f}^{*}\right) \mathbf{e}_{y^{*}}+\frac{\sigma_{e}}{\rho_{\text {ref }}}\left(\mathbf{v}^{*} \times \mathbf{B}^{*}\right) \times \mathbf{B}^{*}$

$\mathbf{v}^{*} \cdot \nabla^{*} T^{*}=\alpha \nabla^{2 *} T^{*}$

$\nabla^{2 *} \mathbf{B}^{*}+\mu_{e} \sigma_{e} \nabla^{*} \times\left(\mathbf{v}^{*} \times \mathbf{B}^{*}\right)=0$

The dimensionless independent and dependent variables are defined as

$\mathrm{X}=\frac{\mathrm{X}^{*}}{\mathrm{~L}}, y=\frac{\mathrm{y}^{*}}{\mathrm{~L}}, \mathbf{v}=\frac{\mathbf{v}^{*}}{v_{0}^{*}}, p=\frac{p^{*}}{\rho_{\text {ref }}\left(v_{0}^{*}\right)^{2}}, \quad T=\frac{T^{*}-T_{r e f}^{*}}{T_{H}^{*}-T_{C}^{*}}, \quad \mathbf{B}=\frac{\mathbf{B}^{*}}{\left|\mathbf{B}_{0}^{*}\right|}$

where $v_{0}^{*}$ denotes a reference velocity for the fluid flow inside the cavity. Therefore, substituting the dimensionless groups given by Eq. (17) into Eqs. (13)-(16), yields

$\nabla \cdot \mathbf{v}=0$

$\mathbf{v} \cdot \nabla \mathbf{v}=-\nabla p+\frac{1}{R e} \nabla^{2} \mathbf{v}+\frac{G r}{R e^{2}} T \mathbf{e}_{y}+\frac{H a^{2}}{R e}(\mathbf{v} \times \mathbf{B}) \times \mathbf{B}$

$\mathbf{v} \cdot \nabla T=\frac{1}{\operatorname{RePr}} \nabla^{2} T$

$\nabla^{2} \mathbf{B}+\operatorname{Re}_{m} \boldsymbol{\nabla} \times(\mathbf{v} \times \mathbf{B})=0$

The following operator identities are readily verified $\nabla^{*}(\bullet)=\mathrm{L}^{-1} \nabla(\bullet)$ and $\nabla^{2 *}(\bullet)=\mathrm{L}^{-2} \nabla^{2}(\bullet)$. Also, the dimensionless groups $\mathrm{Re}, \mathrm{Pr}, \mathrm{Gr}, \mathrm{Ha}$, and $R e_{m}$, respectively, the Reynolds number, the Prandtl number, the Grashof number, the Hartmann number, and the magnetic Reynolds number, are defined as

$\operatorname{Re}=\frac{v_{0}^{*} L}{v}, \quad \operatorname{Pr}=\frac{v}{\alpha}, \quad G r=\frac{g \beta \Delta T L^{3}}{v^{2}}, \quad H a=\left|\mathbf{B}_{0}^{*}\right| L \sqrt{\frac{\sigma_{e}}{\rho_{\text {ref }} v}}, \quad \operatorname{Re}_{m}=\frac{v_{0}^{*} L}{v_{m}}$

(22a-e) 
where $\Delta T^{*} \equiv T_{H}^{*}-T_{C}^{*}$ denotes a characteristic temperature difference and $v_{m} \equiv\left(\mu_{e} \sigma_{e}\right)^{-1}$ is referred to as the magnetic diffusivity. The magnitude of Hartmann number, Ha, expresses the ratio of magnetic to viscous forces acting on the fluid, whilst the magnetic Reynolds number, $R e_{m}$, measures the relative importance of diffusive and convective terms in Eq. (21).

For the special case of very low magnetic Reynolds number $\left(R e_{m} \ll 1\right)$, Eq. (21) simplifies to $\nabla^{2} \mathbf{B} \approx 0$, whose solution, for boundary conditions of the first kind $\mathbf{B}=\mathbf{B}_{0}$ prescribed at the walls of the cavity, is simply $\mathbf{B}(x, y)=\mathbf{B}_{0}$ or, equivalently, $\mathbf{B}^{\prime}(x, y)=\mathbf{0}$. Notice that the low magnetic Reynolds number assumption (or, equivalently, the inductionless approximation) decouples Eqs. (19) and (21). Henceforth, one assumes the low magnetic Reynolds number assumption, which is valid for most engineering applications of MHD flows.

The governing partial differential equations for steady twodimensional MHD natural convection within the cavity illustrated in Fig. 1 may thus be rewritten in scalar form in terms of the primitive field variables $u^{*}\left(x^{*}, y^{*}\right), v^{*}\left(x^{*}, y^{*}\right), p^{*}\left(x^{*}, y^{*}\right)$ and $T^{*}\left(x^{*}, y^{*}\right)$ as follows

$$
\frac{\partial u^{*}}{\partial x^{*}}+\frac{\partial v^{*}}{\partial y^{*}}=0
$$

$u^{*} \frac{\partial u^{*}}{\partial x^{*}}+v^{*} \frac{\partial u^{*}}{\partial y^{*}}=-\frac{1}{\rho_{\text {ref }}} \frac{\partial p^{*}}{\partial x^{*}}+v\left(\frac{\partial^{2} u^{*}}{\partial x^{* 2}}+\frac{\partial^{2} u^{*}}{\partial y^{* 2}}\right)$

$$
-\frac{\sigma_{e}\left|\mathbf{B}_{0}^{*}\right|^{2}}{\rho_{\text {ref }}}\left(u^{*} \sin ^{2} \gamma-v^{*} \sin \gamma \cos \gamma\right)
$$

$$
\begin{aligned}
u^{*} \frac{\partial v^{*}}{\partial x^{*}}+v^{*} \frac{\partial v^{*}}{\partial y^{*}}= & -\frac{1}{\rho_{\text {ref }}} \frac{\partial p^{*}}{\partial y^{*}}+v\left(\frac{\partial^{2} v^{*}}{\partial x^{* 2}}+\frac{\partial^{2} v^{*}}{\partial y^{* 2}}\right)+g \beta\left(T^{*}-T_{r e f}^{*}\right) \\
& +\frac{\sigma_{e}\left|\mathbf{B}_{0}^{*}\right|^{2}}{\rho_{\text {ref }}}\left(u^{*} \sin \gamma \cos \gamma-v^{*} \cos ^{2} \gamma\right)
\end{aligned}
$$

$u^{*} \frac{\partial T^{*}}{\partial x^{*}}+v^{*} \frac{\partial T^{*}}{\partial y^{*}}=\alpha\left(\frac{\partial^{2} T^{*}}{\partial x^{* 2}}+\frac{\partial^{2} T^{*}}{\partial y^{* 2}}\right)$

where the symbol $\gamma$ denotes the inclination angle of the magnetic field $\mathbf{B}_{0}^{*}$ with respect to the $x^{*}$-direction, $0 \leq \gamma \leq 2 \pi$ (see Fig. 1); hence, $B_{0 x}^{*}=\left|\mathbf{B}_{0}^{*}\right| \cos \gamma$ and $B_{0 y}^{*}=\left|\mathbf{B}_{0}^{*}\right| \sin \gamma$. Before proceeding with the integral transformation of Eqs. (23)-(26), one eliminates the pressure field $p^{*}\left(x^{*}, y^{*}\right)$, in the usual manner, by defining the streamfunction $\Psi^{*}\left(x^{*}, y^{*}\right)$ such that $u^{*}\left(x^{*}, y^{*}\right)=\partial \Psi^{*} / \partial y^{*}$ and $v^{*}\left(x^{*}, y^{*}\right)=-\partial \Psi^{*} / \partial x^{*}$.

Differentiating Eqs. (24) and (25) with respect to $y^{*}$ and $x^{*}$, respectively, subtracting the resulting equations and then using both the continuity equation and the definition of the streamfunction, one reduces the previous system of four second-order partial differential equations into the following system of two fourthorder and second-order partial differential equations for $\Psi^{*}\left(x^{*}, y^{*}\right)$ and $T^{*}\left(x^{*}, y^{*}\right)$

$$
\begin{aligned}
\frac{\partial\left(\Psi^{*}, \nabla^{2 *} \Psi^{*}\right)}{\partial\left(y^{*}, \boldsymbol{X}^{*}\right)}= & v \nabla^{4 *} \Psi^{*}-g \beta \frac{\partial T^{*}}{\partial \boldsymbol{x}^{*}} \\
& -\frac{\sigma_{e}\left|\mathbf{B}_{0}^{*}\right|^{2}}{\rho_{\text {ref }}}\left(\frac{\partial^{2} \Psi^{*}}{\partial x^{*^{2}}} \cos ^{2} \gamma+2 \frac{\partial^{2} \Psi^{*}}{\partial x^{*} \partial y^{*}} \sin \gamma \cos \gamma+\frac{\partial^{2} \Psi^{*}}{\partial y^{* 2}} \sin ^{2} \gamma\right)
\end{aligned}
$$

$\frac{\partial \Psi^{*}}{\partial y^{*}} \frac{\partial T^{*}}{\partial x^{*}}-\frac{\partial \Psi^{*}}{\partial x^{*}} \frac{\partial T^{*}}{\partial y^{*}}=\alpha\left(\frac{\partial^{2} T^{*}}{\partial x^{* 2}}+\frac{\partial^{2} T^{*}}{\partial y^{* 2}}\right)$

subjected to the following boundary conditions at the cavity walls
$\Psi^{*}\left(0, y^{*}\right)=0 ; \quad \frac{\partial \Psi^{*}\left(0, y^{*}\right)}{\partial x^{*}}=0 ; \quad T^{*}\left(0, y^{*}\right)=T_{H}^{*}, \quad 0<y^{*}<L$

$(29 a-c)$

$\Psi^{*}\left(L, y^{*}\right)=0 ; \quad \frac{\partial \Psi^{*}\left(L, y^{*}\right)}{\partial x^{*}}=0 ; \quad T^{*}\left(L, y^{*}\right)=T_{C}^{*}, \quad 0<y^{*}<L$

$\Psi^{*}\left(x^{*}, 0\right)=0 ; \quad \frac{\partial \Psi^{*}\left(x^{*}, 0\right)}{\partial y^{*}}=0 ; \quad \frac{\partial T^{*}\left(x^{*}, 0\right)}{\partial y^{*}}=0, \quad 0<x^{*}<L$

$\Psi^{*}\left(x^{*}, L\right)=0 ; \quad \frac{\partial \Psi^{*}\left(x^{*}, L\right)}{\partial y^{*}}=0 ; \quad \frac{\partial T^{*}\left(x^{*}, L\right)}{\partial y^{*}}=0, \quad 0<x^{*}<L$

where $\nabla^{2 *} \Psi$ and $\nabla^{4 *} \Psi$ are the well-known Laplace and biharmonic operators whereas $\partial\left(\Psi^{*}, \nabla^{2 *} \Psi\right) / \partial\left(y^{*}, x^{*}\right)$ is defined as

$$
\begin{aligned}
\frac{\partial\left(\Psi^{*}, \nabla^{2 *} \Psi^{*}\right)}{\partial\left(\boldsymbol{y}^{*}, \boldsymbol{X}^{*}\right)}= & \frac{\partial \Psi^{*}}{\partial \boldsymbol{y}^{*}} \frac{\partial}{\partial \boldsymbol{x}^{*}}\left(\frac{\partial^{2} \Psi^{*}}{\partial \boldsymbol{x}^{* 2}}+\frac{\partial^{2} \Psi^{*}}{\partial \boldsymbol{y}^{* 2}}\right) \\
& -\frac{\partial \Psi^{*}}{\partial \boldsymbol{x}^{*}} \frac{\partial}{\partial \boldsymbol{y}^{*}}\left(\frac{\partial^{2} \Psi^{*}}{\partial \boldsymbol{x}^{* 2}}+\frac{\partial^{2} \Psi^{*}}{\partial \boldsymbol{y}^{* 2}}\right)
\end{aligned}
$$

By defining the dimensionless streamfunction as $\Psi(x, y)=$ $\Psi^{*}\left(x^{*}, y^{*}\right) / v$ and then substituting it into Eqs. (27)-(29), together with the dimensionless variables previously defined, yields

$$
\begin{aligned}
\frac{\partial \Psi}{\partial y}\left(\frac{\partial^{3} \Psi}{\partial x^{3}}+\frac{\partial^{3} \Psi}{\partial x \partial y^{2}}\right)-\frac{\partial \Psi}{\partial x}\left(\frac{\partial^{2} \Psi}{\partial x^{2} \partial y}+\frac{\partial^{3} \Psi}{\partial y^{3}}\right) \\
=\frac{\partial^{4} \Psi}{\partial x^{4}}+2 \frac{\partial^{4} \Psi}{\partial x^{2} \partial y^{2}}+\frac{\partial^{4} \Psi}{\partial y^{4}}-G r \frac{\partial T}{\partial x} \\
\quad-H a^{2}\left(\frac{\partial^{2} \Psi}{\partial x^{2}} \cos ^{2} \gamma+2 \frac{\partial^{2} \Psi}{\partial x \partial y} \sin \gamma \cos \gamma+\frac{\partial^{2} \Psi}{\partial y^{2}} \sin ^{2} \gamma\right)
\end{aligned}
$$

$\frac{\partial \Psi}{\partial y} \frac{\partial T}{\partial x}-\frac{\partial \Psi}{\partial x} \frac{\partial T}{\partial y}=\frac{1}{P r}\left(\frac{\partial^{2} T}{\partial x^{2}}+\frac{\partial^{2} T}{\partial y^{2}}\right)$

with associated dimensionless boundary conditions

$$
\begin{aligned}
& \Psi(0, y)=0 ; \quad \frac{\partial \Psi(0, y)}{\partial x}=0 ; \quad T(0, y)=1 / 2, \quad 0<y<1 \\
& \Psi(1, y)=0 ; \quad \frac{\partial \Psi(1, y)}{\partial x}=0 ; \quad T(1, y)=-1 / 2, \quad 0<y<1
\end{aligned}
$$

$\Psi(x, 0)=0 ; \quad \frac{\partial \Psi(x, 0)}{\partial y}=0 ; \quad \frac{\partial T(x, 0)}{\partial y}=0, \quad 0<x<1$

$\Psi(x, 1)=0 ; \quad \frac{\partial \Psi(x, 1)}{\partial y}=0 ; \quad \frac{\partial T(x, 1)}{\partial y}=0, \quad 0<x<1$

The MHD natural convection problem given by Eqs. (31)-(33) can now be handled by the GITT.

\section{Integral transform solution}

By inspection of Eqs. (33), it is easy to verify that the boundary conditions at the horizontal walls $(y=0$ and $y=1)$ are all homogeneous; on the other hand, there is a non-homogeneous boundary condition for the temperature at the vertical wall $x=0$. In order to improve the convergence of the eigenfunction expansion in the presence of non-homogeneous boundary conditions, a 
common strategy is to split the solution into a filtered potential and a filter, prior to integral transformation. However, here it is possible to progress without filtering, eliminating the $y$ independent variable through integral transformation and leaving the non-homogeneous boundary condition to be solved together with the resulting system of ordinary differential equations for the transformed potentials, as now demonstrated.

The GITT solution of the MHD natural convection problem comprises three steps. The first step is to choose an appropriate eigenvalue problem to provide the eigenvalues and eigenfunctions for the proposed expansions. By inspection of the diffusive terms appearing on Eqs. (31) and (32), the eigenvalue problems chosen for the series expansions of $\Psi(x, y)$ and $T(x, y)$ are, respectively, given by:

$\frac{d^{4} \Phi_{\mathrm{i}}}{d y^{4}}-\alpha_{\mathrm{i}}^{4} \Phi_{\mathrm{i}}(y)=0, \quad 0<y<1$

$\Phi_{\mathrm{i}}(0)=0 ; \quad \frac{d \Phi_{\mathrm{i}}(0)}{d y}=0$

$\Phi_{\mathrm{i}}(1)=0 ; \quad \frac{d \Phi_{\mathrm{i}}(1)}{d y}=0$

and

$\frac{d^{2} \Gamma_{\mathrm{i}}}{d y^{2}}+\beta_{\mathrm{i}}^{2} \Gamma_{\mathrm{i}}(y)=0, \quad 0<y<1$

$\frac{d \Gamma_{\mathrm{i}}(0)}{d y}=0 ; \quad \frac{d \Gamma_{\mathrm{i}}(1)}{d y}=0$

The eigenfunctions, eigenvalues, orthogonality properties and norms for the two eigenvalue problems are analytically determined, as follows:

$\Phi_{i}(y)= \begin{cases}\frac{\cos \left[\alpha_{i}(y-1 / 2)\right]}{\cos \left(\alpha_{i} / 2\right)}-\frac{\cosh \left[\alpha_{i}(y-1 / 2)\right]}{\cosh \left(\alpha_{i} / 2\right)}, & \text { for } i=1,3,5, \ldots \\ \frac{\sin \left[\alpha_{i}(y-1 / 2)\right]}{\sin \left(\alpha_{i} / 2\right)}-\frac{\sinh \left[\alpha_{i}(y-1 / 2)\right]}{\sinh \left(\alpha_{i} / 2\right)}, & \text { for } i=2,4,6, \ldots\end{cases}$

$\tan \left(\alpha_{i} / 2\right)= \begin{cases}-\tanh \left(\alpha_{i} / 2\right), & \text { for } i=1,3,5, \ldots \\ \tanh \left(\alpha_{i} / 2\right), & \text { for } i=2,4,6, \ldots\end{cases}$

$\int_{0}^{1} \Phi_{\mathrm{i}}(y) \Phi_{\mathrm{j}}(y) \mathrm{d} y=\left\{\begin{array}{ll}0, & \text { for } \mathrm{i} \neq \mathrm{j} \\ N_{\Phi_{\mathrm{i}}}, & \text { for } \mathrm{i}=\mathrm{j}\end{array} ; \quad N_{\Phi_{\mathrm{i}}}=\int_{0}^{1} \Phi_{\mathrm{i}}^{2}(y) \mathrm{d} y=1\right.$

(36e-g)

and

$\Gamma_{\mathrm{i}}(y)=\cos \left(\beta_{\mathrm{i}} y\right) ; \quad \beta_{\mathrm{i}}=(\mathrm{i}-1) \pi, \quad$ for $\mathrm{i}=1,2,3, \ldots$

$\int_{0}^{1} \Gamma_{\mathrm{i}}(y) \Gamma_{\mathrm{j}}(y) \mathrm{d} y=\left\{\begin{array}{ll}0, & \text { for } \mathrm{i} \neq \mathrm{j} \\ N_{\Gamma_{\mathrm{i}}}, & \text { for } \mathrm{i}=\mathrm{j}\end{array} ;\right.$

$N_{\Gamma_{\mathrm{i}}}=\int_{0}^{1} \Gamma_{\mathrm{i}}^{2}(y) \mathrm{d} y= \begin{cases}1, & \text { if } \mathrm{i}=1 \\ 1 / 2, & \text { if } \mathrm{i}>1\end{cases}$

$(37 c-f)$

where $\Phi_{\mathrm{i}}$ and $\Gamma_{\mathrm{i}}$ are the eigenfunctions, $\alpha_{\mathrm{i}}$ and $\beta_{\mathrm{i}}$ are the eigenvalues, and $N_{\Phi \mathrm{i}}$ and $N_{\Gamma \mathrm{i}}$ are the norms for the streamfunction and temperature fields, respectively. The eigenvalues $\alpha_{i}$ are the positive real roots of the transcendental equations $(36 \mathrm{c}, \mathrm{d})$. These positive real roots may be readily computed with the aid of an appropriate numerical algorithm for nonlinear algebraic equations. The normalization integrals were computed within the Mathematica symbolic manipulation platform [42] and the closed-form expressions obtained are given by Eqs. (36g) and (37e,f).
The second step comprises the definition of the integral transform pair. The eigenfunctions $\Phi_{\mathrm{i}}(y)$ and $\Gamma_{\mathrm{i}}(y)$ are orthogonal functions that satisfy the orthogonality properties given by Eqs. (36e,f) and $(37 c, d)$, respectively. Hence, we determine the following integral transform pairs for the potentials $\Psi(x, y)$ and $T(x, y)$, respectively, as

$\bar{\Psi}_{\mathrm{i}}(x)=\int_{0}^{1} \tilde{\Phi}_{\mathrm{i}}(y) \Psi(x, y) \mathrm{d} y, \quad$ transform

$\Psi(x, y)=\sum_{\mathrm{i}=1}^{\infty} \tilde{\Phi}_{\mathrm{i}}(y) \bar{\Psi}_{\mathrm{i}}(x), \quad$ inverse

and

$\overline{\mathrm{T}}_{\mathrm{i}}(x)=\int_{0}^{1} \tilde{\Gamma}_{\mathrm{i}}(y) \mathrm{T}(x, y) \mathrm{d} y, \quad$ transform

$\mathrm{T}(x, y)=\sum_{\mathrm{i}=1}^{\infty} \tilde{\Gamma}_{\mathrm{i}}(y) \overline{\mathrm{T}}_{\mathrm{i}}(x), \quad$ inverse

The modified eigenfunctions $\tilde{\Phi}_{\mathrm{i}}(y)$ and $\tilde{\Gamma}_{\mathrm{i}}(y)$ are normalized and related to the original ones as:

$\tilde{\Phi}_{\mathrm{i}}(y) \equiv \Phi_{\mathrm{i}}(y) / N_{\Phi_{\mathrm{i}}}^{1 / 2}=\Phi_{\mathrm{i}} \quad$ and

$\tilde{\Gamma}_{\mathrm{i}}(y)=\Gamma_{\mathrm{i}}(y) / N_{\Gamma_{\mathrm{i}}}^{1 / 2}= \begin{cases}\Gamma_{\mathrm{i}}(y), & \text { if } \mathrm{i}=1 \\ \sqrt{2} \Gamma_{\mathrm{i}}(y), & \text { if } \mathrm{i}>1\end{cases}$

The third step comprises the integral transformation of Eqs. (31) and (32). For this purpose, Eqs. (31) and (32), followed by the boundary conditions in the $x$ direction given by Eqs. (33a-f), are multiplied respectively by $\tilde{\Phi}_{\mathrm{i}}(y)$ and $\tilde{\Gamma}_{\mathrm{i}}(y)$ and integrated over the domain $[0,1]$ in the $y$ direction, and the inverse formulae given by Eqs. (38b) and (39b) are employed. After the usual manipulations, the following nonlinear coupled ordinary differential system is obtained for the computation of the transformed potentials $\bar{\Psi}_{\mathrm{i}}(x)$ and $\overline{\mathrm{T}}_{\mathrm{i}}(x)$, respectively:

$$
\begin{aligned}
\frac{d^{4} \bar{\Psi}_{\mathrm{i}}}{d x^{4}}= & -\alpha_{\mathrm{i}}^{4} \bar{\Psi}_{\mathrm{i}}(x)+H a^{2} \cos ^{2} \gamma \frac{d^{2} \bar{\Psi}_{\mathrm{i}}}{d x^{2}} \\
& -\sum_{\mathrm{j}=1}^{\infty}\left\{2 A_{\mathrm{ij}} \frac{d^{2} \bar{\Psi}_{\mathrm{j}}}{d x^{2}}-H a^{2}\left[\sin ^{2} \gamma \mathrm{A}_{\mathrm{ij}} \bar{\Psi}_{\mathrm{j}}(x)+2 \sin \gamma \cos \gamma \mathrm{B}_{\mathrm{ij}} \frac{d \bar{\Psi}_{\mathrm{j}}}{d x}\right]\right\} \\
& -\sum_{\mathrm{j}=1}^{\infty} \sum_{\mathrm{k}=1}^{\infty}\left[\mathrm{C}_{\mathrm{ijk}} \frac{d \bar{\Psi}_{\mathrm{j}}}{d x} \bar{\Psi}_{\mathrm{k}}(x)+\mathrm{D}_{\mathrm{ijk}} \frac{d \bar{\Psi}_{\mathrm{j}}}{d x} \frac{d^{2} \bar{\Psi}_{\mathrm{k}}}{d x^{2}}\right. \\
& \left.-\mathrm{E}_{\mathrm{ijk}} \bar{\Psi}_{\mathrm{j}} \frac{d \bar{\Psi}_{\mathrm{k}}}{d x}-\mathrm{D}_{\mathrm{ijk}} \frac{d^{3} \bar{\Psi}_{\mathrm{j}}}{d x^{3}} \bar{\Psi}_{\mathrm{k}}(x)\right]+\mathrm{Gr} \sum_{\mathrm{j}=1}^{\infty} \mathrm{F}_{\mathrm{ij}} \frac{d \overline{\mathrm{T}}_{\mathrm{j}}}{d x} \\
\frac{d^{2} \overline{\mathrm{T}}_{\mathrm{i}}}{d x^{2}}= & \beta_{\mathrm{i}}^{2} \overline{\mathrm{T}}_{\mathrm{i}}(x)-\operatorname{Pr} \sum_{\mathrm{j}=1}^{\infty} \sum_{\mathrm{k}=1}^{\infty}\left[\mathrm{G}_{\mathrm{ijk}} \overline{\mathrm{T}}_{\mathrm{j}}(x) \frac{d \bar{\Psi}_{\mathrm{k}}}{d x}-\mathrm{H}_{\mathrm{ijk}} \frac{d \overline{\mathrm{T}}_{\mathrm{j}}}{d x} \bar{\Psi}_{\mathrm{k}}(x)\right] \\
\bar{\Psi}_{\mathrm{i}}(0)= & 0 ; \frac{d \bar{\Psi}_{\mathrm{i}}(0)}{d x}=0 ; \overline{\mathrm{T}}_{\mathrm{i}}(0)=\overline{\mathrm{f}}_{\mathrm{i}} / 2 \\
\bar{\Psi}_{\mathrm{i}}(1)= & 0 ; \frac{d \bar{\Psi}_{\mathrm{i}}(1)}{d x}=0 ; \overline{\mathrm{T}}_{\mathrm{i}}(1)=-\overline{\mathrm{f}}_{\mathrm{i}} / 2
\end{aligned}
$$

where

$$
\begin{aligned}
& \mathrm{A}_{\mathrm{ij}}=\int_{0}^{1} \tilde{\Phi}_{\mathrm{i}}(y) \tilde{\Phi}_{\mathrm{j}}^{\prime \prime}(y) \mathrm{d} y ; \quad \mathrm{B}_{\mathrm{ij}}=\int_{0}^{1} \tilde{\Phi}_{\mathrm{i}}(y) \tilde{\Phi}_{\mathrm{j}}^{\prime}(y) \mathrm{d} y ; \\
& \mathrm{C}_{\mathrm{ijk}}=\int_{0}^{1} \tilde{\Phi}_{\mathrm{i}}(y) \tilde{\Phi}_{\mathrm{j}}(y) \tilde{\Phi}_{\mathrm{k}}^{\prime \prime \prime}(y) \mathrm{d} y
\end{aligned}
$$




$$
\begin{aligned}
& \mathrm{D}_{\mathrm{ijk}}=\int_{0}^{1} \tilde{\Phi}_{\mathrm{i}}(y) \tilde{\Phi}_{\mathrm{j}}(y) \tilde{\Phi}_{\mathrm{k}}^{\prime}(y) \mathrm{d} y ; \\
& \mathrm{E}_{\mathrm{ijk}}=\int_{0}^{1} \tilde{\Phi}_{\mathrm{i}}(y) \tilde{\Phi}_{\mathrm{j}}^{\prime}(y) \tilde{\Phi}_{\mathrm{k}}^{\prime \prime}(y) \mathrm{d} y ; \quad \mathrm{F}_{\mathrm{ij}}=\int_{0}^{1} \tilde{\Phi}_{\mathrm{i}}(y) \tilde{\Gamma}_{\mathrm{j}}(y) \mathrm{d} y
\end{aligned}
$$

$\mathrm{G}_{\mathrm{ijk}}=\int_{0}^{1} \tilde{\Gamma}_{\mathrm{i}}(y) \tilde{\Gamma}_{\mathrm{j}}^{\prime}(y) \tilde{\Phi}_{\mathrm{k}}(y) \mathrm{d} y ;$

$\mathrm{H}_{\mathrm{ijk}}=\int_{0}^{1} \tilde{\Gamma}_{\mathrm{i}}(y) \tilde{\Gamma}_{\mathrm{j}}(y) \tilde{\Phi}_{\mathrm{k}}^{\prime}(y) \mathrm{d} y ; \quad \overline{\mathrm{f}}_{\mathrm{i}}=\int_{0}^{1} \tilde{\Gamma}_{\mathrm{i}}(y) \mathrm{d} y$

The infinite series expansions for $\Psi(x, y)$ and $T(x, y)$ are henceforth truncated to a finite number of terms, denoted respectively by $N_{\Psi}$ and $N_{T}$. The above integral coefficients appearing in the coupled system of ordinary differential equations for the transformed potentials, $\bar{\Psi}_{i}(x)$ and $\bar{T}_{i}(x)$, are evaluated by symbolic computation within the Mathematica platform [42], and closed-form expressions are obtained.

The coupled system of ordinary differential equations has been numerically solved with two Fortran subroutines, namely: (i) subroutine BVPFD from the IMSL Library [43] and (ii) Fortran subroutine BVP_SOLVER developed by Shampine et al. [44] and freely available for download at http://cs.stmarys.ca/ muir/BVP_SOLVER_Webpage.shtml. A dedicated Fortran 90 code has been developed to perform the computations described in the next section. The relative error target adopted for the two previously mentioned Fortran subroutines was set to $10^{-4}$. The final results achieved by the two subroutines were critically compared, and it was verified that they were coincident to the fourth significant digit, thus confirming the automatic relative error control in both algorithms.

Once the transformed potentials are obtained, heat transfer parameters such as Nusselt numbers may be computed. Therefore, the local and average Nusselt numbers are defined as

$$
\begin{aligned}
N u & =u(x, y) T(x, y)-\frac{\partial T(x, y)}{\partial x} \\
& =\frac{\partial \Psi(x, y)}{\partial y} T(x, y)-\frac{\partial T(x, y)}{\partial x} ; \quad \overline{N u}_{x}=\int_{0}^{1} N u \mathrm{dy}
\end{aligned}
$$

After introducing the inverse formulae given by Eqs. (38b) and (39b), it results

$$
\begin{aligned}
& N u=\sum_{\mathrm{i}=1}^{\infty} \sum_{\mathrm{j}=1}^{\infty} \tilde{\Phi}_{\mathrm{i}}^{\prime}(y) \tilde{\Gamma}_{\mathrm{j}}(y) \bar{\Psi}_{\mathrm{i}}(x) \overline{\mathrm{T}}_{\mathrm{j}}(x)-\sum_{\mathrm{i}=1}^{\infty} \tilde{\Gamma}_{\mathrm{i}}(y) \frac{d \overline{\mathrm{T}}_{\mathrm{i}}(x)}{d x} \\
& \overline{N u}_{\mathrm{x}}=\sum_{\mathrm{i}=1}^{\infty} \sum_{\mathrm{j}=1}^{\infty} \mathrm{I}_{\mathrm{ij}} \bar{\Psi}_{\mathrm{i}}(x) \overline{\mathrm{T}}_{\mathrm{j}}(x)-\sum_{\mathrm{i}=1}^{\infty} \overline{\mathrm{f}}_{\mathrm{i}} \frac{d \overline{\mathrm{T}}_{\mathrm{i}}(x)}{d x} ; \quad \mathrm{I}_{\mathrm{ij}}=\int_{0}^{1} \tilde{\Phi}_{\mathrm{i}}^{\prime}(y) \tilde{\Gamma}_{\mathrm{j}}(y) \mathrm{dy}
\end{aligned}
$$

The local and average Nusselt numbers at the hot vertical wall are simply computed by substituting $x=0$ into Eqs. (44).

\section{Results and discussions}

In order to evaluate the accuracy and robustness of the proposed eigenfunction expansion approach, several test cases were analyzed with the developed Fortran code. The numerical results obtained for the velocity and temperature distributions, as well as for both local and average Nusselt numbers at the vertical walls are reported, discussed and, whenever possible, critically compared against previous ones reported in [7-10,14].

The computations were performed for two Grashof and three Rayleigh numbers, $\mathrm{Gr}=\left\{10^{4}, 10^{6}\right\}$ and $\mathrm{Ra}=\left\{10^{4}, 10^{5}, 10^{6}\right\}$, for Hartmann numbers $\mathrm{Ha} \in[0,300]$, for two Prandtl numbers, $\operatorname{Pr}=\{0.71,0.733\}$, and for magnetic field inclination angles

\begin{tabular}{|c|c|c|c|c|c|c|c|c|c|c|c|c|c|c|}
\hline \multirow[t]{2}{*}{$N_{\Psi} / N_{T}$} & \multicolumn{14}{|l|}{$\mathbf{H a}=\mathbf{0} \Psi(x, y)$} \\
\hline & $x=0.1 ; y=0.1$ & $x=0.1 ; y=0.5$ & $x=0.1 ; y=0.9$ & $x=0.5 ; y=0.1$ & \multicolumn{2}{|c|}{$x=0.5 ; y=0.5$} & \multicolumn{2}{|c|}{$x=0.5 ; y=0.9$} & \multicolumn{2}{|c|}{$x=0.9 ; y=0.1$} & \multicolumn{2}{|c|}{$x=0.9 ; y=0.5$} & \multicolumn{2}{|c|}{$x=0.9 ; y=0.9$} \\
\hline $10 / 10$ & -0.3526 & -1.381 & -0.1787 & -0.9444 & -6.146 & & -0.9444 & & -0.1787 & & -1.381 & & -0.352 & \\
\hline $20 / 20$ & -0.3520 & -1.380 & -0.1791 & -0.9442 & -6.146 & & -0.9442 & & -0.1791 & & -1.380 & & -0.352 & \\
\hline $30 / 30$ & -0.3521 & -1.380 & -0.1791 & -0.9442 & -6.146 & & -0.9442 & & -0.1791 & & -1.380 & & -0.352 & \\
\hline $40 / 40$ & -0.3521 & -1.380 & -0.1791 & -0.9442 & -6.146 & & -0.9442 & & -0.1791 & & -1.380 & & -0.352 & \\
\hline \multirow[t]{2}{*}{$N_{\Psi} / N_{T}$} & \multicolumn{13}{|l|}{$T(x, y)+1 / 2$} & \multirow[t]{2}{*}{$\overline{N u}_{\mathrm{x}=0}$} \\
\hline & $x=0.1 ; y=0.1$ & $x=0.1 ; y=0.5$ & $x=0.1 ; y=0.9$ & $x=0.5 ; y=0.1$ & $x=0.5 ; y=0.5$ & \multicolumn{2}{|c|}{$x=0.5 ; y=0.9$} & \multicolumn{2}{|c|}{$x=0.9 ; y=0.1$} & \multicolumn{2}{|c|}{$x=0.9 ; y=0.5$} & \multicolumn{2}{|c|}{$x=0.9 ; y=0.9$} & \\
\hline $10 / 10$ & 0.6963 & 0.7905 & 0.9388 & 0.2284 & 0.5000 & \multicolumn{2}{|c|}{0.7716} & \multicolumn{2}{|c|}{0.06121} & \multicolumn{2}{|c|}{0.2095} & \multicolumn{2}{|l|}{0.3037} & 2.010 \\
\hline $20 / 20$ & 0.6956 & 0.7903 & 0.9387 & 0.2282 & 0.5000 & \multicolumn{2}{|c|}{0.7718} & \multicolumn{2}{|c|}{0.06129} & \multicolumn{2}{|c|}{0.2097} & \multicolumn{2}{|l|}{0.3044} & 2.011 \\
\hline $30 / 30$ & 0.6956 & 0.7903 & 0.9387 & 0.2282 & 0.5000 & 0.7 & 18 & 0.06 & 28 & 0.209 & & 0.3044 & & 2.011 \\
\hline $40 / 40$ & 0.6956 & 0.7903 & 0.9387 & 0.2282 & 0.5000 & 0.7 & 18 & 0.06 & 28 & 0.209 & & 0.3044 & & 2.011 \\
\hline$N_{\Psi} / N_{T}$ & $\mathbf{H a}=\mathbf{5 0} \Psi(x, y)$ & & & & & & & & & & & & & \\
\hline & $x=0.1 ; y=0.1$ & $x=0.1 ; y=0.5$ & $x=0.1 ; y=0.9$ & $x=0.5 ; y=0.1$ & $x=0.5 ; y=$ & 0.5 & $x=0.5 ; y=$ & 0.9 & $x=0.9 ; y$ & $=0.1$ & $x=0.9$ & $y=0.5$ & $x=0.9$ & $y=0.9$ \\
\hline $10 / 10$ & -0.07250 & -0.1436 & -0.06060 & -0.1896 & -0.4700 & & -0.1896 & & -0.06060 & & -0.1436 & & -0.072 & 50 \\
\hline $20 / 20$ & -0.07228 & -0.1430 & -0.06050 & -0.1894 & -0.4693 & & -0.1894 & & -0.06050 & & -0.1430 & & -0.072 & 28 \\
\hline $30 / 30$ & -0.07233 & -0.1431 & -0.06053 & -0.1895 & -0.4693 & & -0.1895 & & -0.06053 & & -0.1431 & & -0.072 & 33 \\
\hline $40 / 40$ & -0.07232 & -0.1431 & -0.06053 & -0.1895 & -0.4693 & & -0.1895 & & -0.06053 & & -0.1431 & & -0.072 & 32 \\
\hline$N_{\Psi} / N_{T}$ & $T(x, y)+1 / 2$ & & & & & & & & & & & & & $\overline{N u}_{\mathrm{x}=0}$ \\
\hline & $x=0.1 ; y=0.1$ & $x=0.1 ; y=0.5$ & $x=0.1 ; y=0.9$ & $x=0.5 ; y=0.1 \quad x$ & $x=0.5 ; y=0.5$ & $x=$ & $.5 ; y=0.9$ & $x=0$ & $9 ; y=0.1$ & $x=0$ & $y=0.5$ & $x=0.9$ & $y=0.9$ & \\
\hline $10 / 10$ & 0.8784 & 0.8988 & 0.9168 & 0.4391 & 0.5000 & 0.5 & & 0.08 & 18 & 0.10 & & 0.1217 & & 1.019 \\
\hline $20 / 20$ & 0.8783 & 0.8988 & 0.9168 & 0.4390 & 0.5000 & 0.5 & 10 & 0.08 & 16 & 0.101 & & 0.1217 & & 1.019 \\
\hline $30 / 30$ & 0.8783 & 0.8988 & 0.9168 & 0.4390 & 0.5000 & 0.5 & & 0.08 & & 0.10 & & 0.1217 & & 1.019 \\
\hline $40 / 40$ & 0.8783 & 0.8988 & 0.9168 & 0.4390 & 0.5000 & 0.5 & & 0.08 & 17 & 0.10 & & 0.1217 & & 1.019 \\
\hline
\end{tabular}
$\gamma \in\left[-90^{\circ}, 90^{\circ}\right]$ in order to allow for comparisons with the literature. The above Grashof and Rayleigh numbers cover widely

Table 1

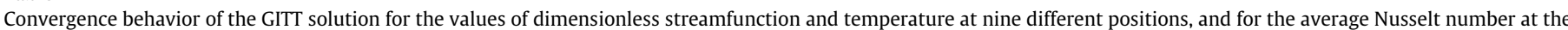
hot vertical wall $\left(\overline{N u}_{\mathrm{x}=0}\right)$ for $\mathrm{Gr}=10^{4}, \mathrm{Ha}=0$ and 50, $\gamma=0^{\circ}$ and $\mathrm{Pr}=0.71$. 
different intensities of buoyancy-driven flows. It is important to mention that the reference temperature was taken as the mean between the temperatures of the hot and cold walls, i.e., $T_{r e f}^{*}=\left(T_{H}\right.$ $\left.*+T_{C}^{*}\right) / 2$. Therefore, in order to allow for comparisons with previous works in the literature, the dimensionless temperature is computed by adding $1 / 2$, i.e., $T(x, y)+1 / 2$. The Hartmann numbers analyzed cover the range from extremely low to high magnetic field strengths.

\subsection{Convergence}

In the first set of results, the convergence of the GITT solution is analyzed for the values of the dimensionless streamfunction and temperature at nine different positions $\left(x_{r}, y_{s}\right)$, with $x_{r} \in\{0.1,0.5,0.9\}$ and $y_{s} \in\{0.1,0.5,0.9\}$, and for the average Nusselt number at the hot vertical wall, $\overline{N u}_{\mathrm{x}=0}$. Table 1 reports the numerical results obtained for $\mathrm{Gr}=10^{4}, \gamma=0^{\circ}$ and $\mathrm{Pr}=0.71$, for increasing truncation orders $N_{\Psi}$ and $N_{T}$, respectively, for $\mathrm{Ha}=0$ and $\mathrm{Ha}=50$. Table 2 reports similar results for a two orders-of-magnitude higher Grashof number $\left(\mathrm{Gr}=10^{6}\right)$. Truncation orders are increased up to 50 for the higher Grashof number in order to achieve convergence to at least three significant digits. The results reported in the previous tables for the streamfunction and temperature were computed for equal truncation orders $N_{\Psi}$ and $N_{\text {T }}$. Hence, it is not possible from the outputs in Tables 1 and 2 to identify which potential (streamfunction or temperature) has the strongest influence in the rate of convergence of the GITT solution. In this context, Table 3 provides a convergence analysis, again for dimensionless streamfunction and temperature at the same nine different positions, for $\mathrm{Gr}=10^{6}, \mathrm{Ha}=100, \gamma=0^{\circ}$ and $\mathrm{Pr}=0.71$, for two cases: (a) for a fixed truncation order in the streamfunction expansion, $N_{\Psi}=50$, but with increasing truncation order in the temperature expansion, $N_{T}$; (b) for a fixed truncation order in the temperature expansion, $N_{\mathrm{T}}=50$, but with increasing truncation order in the streamfunction expansion, $N_{\Psi}$. From the analysis of Tables $1-3$, we may draw the following important conclusions. Firstly, for the lower Grashof number, convergence to three significant digits is achieved already with $N_{\Psi}=N_{T}=20$. Secondly, for the higher Grashof number, convergence to three significant digits is attained with higher truncation orders $\left(N_{\Psi}=N_{T}=30\right)$, as expected, because larger temperature and velocity gradients are induced by larger buoyancy forces. Thirdly, as the Hartmann number increases, higher truncation orders are required to achieve the same accuracy (three significant digits). Fourthly, the convergence for the temperature field is noticeably faster than that for the streamfunction. Fifthly, convergence is slower for the points $(0.1,0.1)$ and $(0.9,0.9)$ which are closer to regions with larger temperature and velocity gradients. It can be concluded from the convergence analysis in Table 3 that the computational cost can be further reduced by considering different truncation orders for the two fields, since smaller truncation orders might be sufficient for full convergence, as can observed from fixing one of them. Based on the results reported in Table 3, it is also concluded that for a fixed high truncation order $N_{\Psi}$ accurate results are achieved for both streamfunction and temperature with lower truncation orders $N_{T}$. On the other hand, for a fixed $N_{T}$, accurate results for the streamfunction are achieved with high truncation orders $N_{\Psi}$, although much lower truncation orders $N_{\Psi}$ are required for both the temperature and average Nusselt number at the hot wall. To sum up, the temperature potential requires a lower truncation order than the streamfunction; therefore, we may conclude that the latter is mainly responsible for the delay in the convergence speed of the GITT solution. Finally, in order to

Table 2

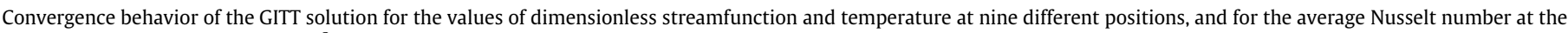
hot vertical wall $\left(\overline{N u}_{\mathrm{x}=0}\right)$ for $\mathrm{Gr}=10^{6}, \mathrm{Ha}=0$ and $50, \gamma=0^{\circ}$ and $\operatorname{Pr}=0.71$.

\begin{tabular}{|c|c|c|c|c|c|c|c|c|c|c|c|c|c|c|c|}
\hline \multirow[t]{2}{*}{$N_{\Psi} / N_{T}$} & \multicolumn{15}{|l|}{$\mathbf{H a}=\mathbf{0} \Psi(x, y)$} \\
\hline & $x=0.1 ; y=0.1$ & $x=0.1 ; y=0.5$ & $x=0.1 ; y=0.9$ & \multicolumn{2}{|l|}{$x=0.5 ; y=0.1$} & \multicolumn{2}{|c|}{$x=0.5 ; y=0.5$} & \multicolumn{2}{|c|}{$x=0.5 ; y=0.9$} & \multicolumn{2}{|c|}{$x=0.9 ; y=0.1$} & \multicolumn{2}{|c|}{$x=0.9 ; y=0.5$} & \multicolumn{2}{|c|}{$x=0.9 ; y=0.9$} \\
\hline $10 / 10$ & -5.515 & -18.89 & -7.944 & -4.103 & & -21.04 & & -4.103 & & -7.944 & & -18.89 & & -5.515 & \\
\hline $20 / 20$ & -5.738 & -18.95 & -7.974 & -4.076 & & -21.19 & & -4.076 & & -7.974 & & -18.95 & & -5.738 & \\
\hline $30 / 30$ & -5.749 & -18.96 & -7.996 & -4.078 & & -21.20 & & -4.078 & & -7.996 & & -18.96 & & -5.749 & \\
\hline $40 / 40$ & -5.750 & -18.96 & -7.994 & -4.078 & & -21.20 & & -4.078 & & -7.994 & & -18.96 & & -5.750 & \\
\hline $50 / 50$ & -5.750 & -18.96 & -7.995 & -4.078 & & -21.20 & & -4.078 & & -7.995 & & -18.96 & & -5.750 & \\
\hline \multirow[t]{2}{*}{$N_{\Psi} / N_{T}$} & \multicolumn{14}{|l|}{$T(x, y)+1 / 2$} & \multirow{2}{*}{$\overline{N u}_{\mathrm{x}=0}$} \\
\hline & $x=0.1 ; y=0.1$ & $x=0.1 ; y=0.5$ & $x=0.1 ; y=0.9$ & $x=0.5 ; y=0.1$ & \multicolumn{2}{|c|}{$x=0.5 ; y=0.5$} & \multicolumn{2}{|c|}{$x=0.5 ; y=0.9$} & \multicolumn{2}{|c|}{$x=0.9 ; y=0.1$} & \multicolumn{2}{|c|}{$x=0.9 ; y=0.5$} & \multicolumn{2}{|c|}{$x=0.9 ; y=0.9$} & \\
\hline $10 / 10$ & 0.2346 & 0.4896 & 0.8244 & 0.1802 & 0.50 & 000 & 0.81 & 198 & 0.17 & & 0.510 & & 0.7655 & & 7.976 \\
\hline $20 / 20$ & 0.2311 & 0.4875 & 0.8203 & 0.1790 & 0.5 & 000 & 0.82 & 210 & 0.17 & & 0.512 & & 0.7690 & & 8.013 \\
\hline $30 / 30$ & 0.2313 & 0.4876 & 0.8200 & 0.1790 & 0.5 & 000 & 0.82 & 210 & 0.18 & & 0.512 & & 0.7688 & & 8.015 \\
\hline $40 / 40$ & 0.2312 & 0.4876 & 0.8201 & 0.1790 & 0.5 & 000 & 0.82 & 210 & 0.18 & & 0.512 & & 0.7688 & & 8.016 \\
\hline $50 / 50$ & 0.2312 & 0.4876 & 0.8200 & 0.1790 & 0.5 & 000 & 0.82 & 210 & 0.18 & & 0.512 & & 0.7688 & & 8.016 \\
\hline \multirow[t]{2}{*}{$N_{\Psi} / N_{T}$} & \multicolumn{15}{|l|}{$\mathbf{H a}=\mathbf{5 0} \Psi(x, y)$} \\
\hline & $x=0.1 ; y=0.1$ & $x=0.1 ; y=0.5$ & $x=0.1 ; y=0.9$ & \multicolumn{2}{|l|}{$x=0.5 ; y=0.1$} & \multicolumn{2}{|c|}{$x=0.5 ; y=0.5$} & \multicolumn{2}{|c|}{$x=0.5 ; y=0.9$} & \multicolumn{2}{|c|}{$x=0.9 ; y=0.1$} & \multicolumn{2}{|c|}{$x=0.9 ; y=0.5$} & \multicolumn{2}{|c|}{$x=0.9 ; y=0.9$} \\
\hline $10 / 10$ & -4.877 & -8.818 & -2.385 & -4.505 & & -13.04 & & -4.505 & & -2.385 & & -8.818 & & -4.877 & \\
\hline $20 / 20$ & -4.908 & -8.789 & -2.383 & -4.520 & & -13.06 & & -4.520 & & -2.383 & & -8.789 & & -4.908 & \\
\hline $30 / 30$ & -4.921 & -8.793 & -2.386 & -4.522 & & -13.06 & & -4.522 & & -2.386 & & -8.793 & & -4.921 & \\
\hline $40 / 40$ & -4.920 & -8.793 & -2.386 & -4.522 & & -13.06 & & -4.522 & & -2.386 & & -8.793 & & -4.920 & \\
\hline $50 / 50$ & -4.921 & -8.793 & -2.386 & -4.522 & & -13.06 & & -4.522 & & -2.386 & & -8.793 & & -4.921 & \\
\hline \multirow[t]{2}{*}{$N_{\Psi} / N_{T}$} & $T(x, y)+1 / 2$ & & & & & & & & & & & & & & $\overline{N u}_{\mathrm{x}=0}$ \\
\hline & $x=0.1 ; y=0.1$ & $x=0.1 ; y=0.5$ & $x=0.1 ; y=0.9$ & $x=0.5 ; y=0.1$ & $x=$ & $0.5 ; y=0.5$ & $x=0$ & $0.5 ; y=0.9$ & $x=0$ & $9 ; y=0.1$ & $x=0$ & $9 ; y=0.5$ & $x=0.9$ & $y=0.9$ & \\
\hline $10 / 10$ & 0.2594 & 0.6478 & 0.9356 & 0.1619 & $0.5 c$ & 000 & 0.83 & & 0.06 & 442 & 0.352 & & 0.7406 & & 5.451 \\
\hline $20 / 20$ & 0.2578 & 0.6471 & 0.9351 & 0.1615 & 0.5 & 000 & 0.83 & 385 & 0.06 & 488 & 0.352 & & 0.7422 & & 5.465 \\
\hline $30 / 30$ & 0.2586 & 0.6473 & 0.9352 & 0.1616 & 0.5 & 000 & 0.83 & 385 & 0.06 & 481 & 0.352 & & 0.7414 & & 5.466 \\
\hline $40 / 40$ & 0.2585 & 0.6473 & 0.9352 & 0.1616 & 0.5 & 000 & 0.83 & & 0.06 & 485 & 0.352 & & 0.7415 & & 5.466 \\
\hline $50 / 50$ & 0.2585 & 0.6473 & 0.9352 & 0.1616 & 0.5 & 000 & 0.83 & & 0.06 & 483 & 0.352 & & 0.7415 & & 5.466 \\
\hline
\end{tabular}


Table 3

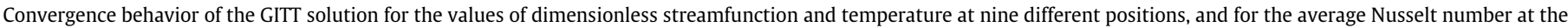

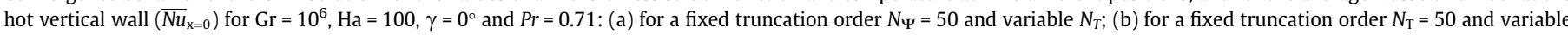
$N$ Y.

\begin{tabular}{|c|c|c|c|c|c|c|c|c|c|c|c|c|c|c|c|}
\hline \multirow[t]{2}{*}{$N_{\Psi} / N_{T}$} & \multicolumn{15}{|l|}{$\Psi(x, y)$} \\
\hline & $x=0.1 ; y=0.1$ & $x=0.1 ; y=0.5$ & $x=0.1 ; y=0.9$ & \multicolumn{2}{|c|}{$x=0.5 ; y=0.1$} & \multicolumn{2}{|c|}{$x=0.5 ; y=0.5$} & \multicolumn{2}{|c|}{$x=0.5 ; y=0.9$} & \multicolumn{2}{|c|}{$x=0.9 ; y=0.1$} & \multicolumn{2}{|c|}{$x=0.9 ; y=0.5$} & \multicolumn{2}{|c|}{$x=0.9 ; y=0.9$} \\
\hline \multicolumn{16}{|c|}{ (a) Fixed $N_{\Psi}$ and variable $N_{T}$} \\
\hline $50 / 10$ & -3.097 & -3.416 & -1.133 & -3.224 & \multicolumn{3}{|c|}{-7.188} & -3.224 & & \multicolumn{2}{|l|}{-1.133} & \multicolumn{2}{|l|}{-3.416} & \multicolumn{2}{|l|}{-3.097} \\
\hline $50 / 20$ & -3.093 & -3.417 & -1.133 & -3.224 & & \multicolumn{2}{|l|}{-7.188} & -3.224 & & \multicolumn{2}{|l|}{-1.133} & \multicolumn{2}{|l|}{-3.417} & \multicolumn{2}{|l|}{-3.093} \\
\hline $50 / 30$ & -3.094 & -3.417 & -1.133 & -3.224 & & & -3.224 & & \multicolumn{2}{|l|}{-1.133} & \multicolumn{2}{|l|}{-3.417} & \multicolumn{2}{|l|}{-3.094} \\
\hline $50 / 40$ & -3.094 & -3.417 & -1.133 & -3.224 & & \multicolumn{2}{|l|}{-7.189} & -3.224 & & \multicolumn{2}{|l|}{-1.133} & \multicolumn{2}{|l|}{-3.417} & \multicolumn{2}{|l|}{-3.094} \\
\hline $50 / 50$ & -3.094 & -3.417 & -1.133 & -3.224 & & -7.189 & & -3.224 & & -1.133 & & -3.417 & & -3.094 & \\
\hline$N_{\Psi} / N_{T}$ & $T(x, y)+1 / 2$ & & & & & & & & & & & & & & $\overline{N u}_{\mathrm{x}=0}$ \\
\hline & $x=0.1 ; y=0.1$ & $x=0.1 ; y=0.5$ & $x=0.1 ; y=0.9$ & $x=0.5 ; y=0.1$ & $x=$ & $0.5 ; y=0.5$ & $x=0$ & $.5 ; y=0.9$ & $x=0$ & $9 ; y=0.1$ & $x=0.9$ & $; y=0.5$ & $x=0.9$ & $y=0.9$ & \\
\hline $50 / 10$ & 0.4114 & 0.7897 & 0.9525 & 0.1772 & 0.50 & 000 & 0.82 & & 0.04 & 752 & 0.210 & & 0.5886 & & 3.213 \\
\hline $50 / 20$ & 0.4086 & 0.7891 & 0.9523 & 0.1767 & 0.50 & 000 & 0.82 & & 0.04 & 773 & 0.210 & & 0.5914 & & 3.213 \\
\hline $50 / 30$ & 0.4096 & 0.7894 & 0.9524 & 0.1768 & 0.50 & 000 & 0.82 & & 0.04 & 757 & 0.210 & & 0.5904 & & 3.214 \\
\hline $50 / 40$ & 0.4094 & 0.7893 & 0.9524 & 0.1768 & 0.50 & 000 & 0.82 & & 0.04 & 763 & 0.210 & & 0.5906 & & 3.214 \\
\hline $50 / 50$ & 0.4095 & 0.7893 & 0.9524 & 0.1768 & 0.50 & 000 & 0.82 & & 0.04 & 760 & 0.210 & & 0.5905 & & 3.214 \\
\hline$N_{\Psi} / N_{T}$ & $\Psi(x, y)$ & & & & & & & & & & & & & & \\
\hline & $x=0.1 ; y=0.1$ & $x=0.1 ; y=0.5$ & $x=0.1 ; y=0.9$ & $x=0.5 ; y=0$ & & $x=0.5 ; y=0$ & 0.5 & $x=0.5 ; y$ & $=0.9$ & $x=0.9 ; y$ & $=0.1$ & $x=0.9$ & $y=0.5$ & $x=0.9$ & $y=0.9$ \\
\hline (b) Fixed & $N_{T}$ and variable & $N_{\Psi}$ & & & & & & & & & & & & & \\
\hline $10 / 50$ & -3.095 & -3.457 & -1.131 & -3.217 & & -7.203 & & -3.217 & & -1.131 & & -3.457 & & -3.095 & \\
\hline $20 / 50$ & -3.086 & -3.414 & -1.133 & -3.223 & & -7.188 & & -3.223 & & -1.133 & & -3.414 & & -3.086 & \\
\hline $30 / 50$ & -3.095 & -3.418 & -1.134 & -3.224 & & -7.189 & & -3.224 & & -1.134 & & -3.418 & & -3.095 & \\
\hline $40 / 50$ & -3.093 & -3.417 & -1.133 & -3.224 & & -7.189 & & -3.224 & & -1.133 & & -3.417 & & -3.093 & \\
\hline $50 / 50$ & -3.094 & -3.417 & -1.133 & -3.224 & & -7.189 & & -3.224 & & -1.133 & & -3.417 & & -3.094 & \\
\hline$N_{\Psi} / N_{T}$ & $T(x, y)+1 / 2$ & & & & & & & & & & & & & & $\overline{N u}_{\mathrm{x}=0}$ \\
\hline & $x=0.1 ; y=0.1$ & $x=0.1 ; y=0.5$ & $x=0.1 ; y=0.9$ & $x=0.5 ; y=0.1$ & $x=$ & $0.5 ; y=0.5$ & $x=0$ & $.5 ; y=0.9$ & $x=0$ & $9 ; y=0.1$ & $x=0.9$ & $; y=0.5$ & $x=0.9$ & $y=0.9$ & \\
\hline $10 / 50$ & 0.3995 & 0.7887 & 0.9527 & 0.1765 & 0.50 & 000 & 0.82 & & 0.04 & 727 & 0.211 & & 0.6006 & & 3.210 \\
\hline $20 / 50$ & 0.4101 & 0.7893 & 0.9524 & 0.1768 & 0.50 & 000 & 0.82 & & 0.04 & 762 & 0.210 & & 0.5899 & & 3.213 \\
\hline $30 / 50$ & 0.4094 & 0.7893 & 0.9524 & 0.1768 & 0.50 & 000 & 0.82 & & 0.04 & 760 & 0.210 & & 0.5906 & & 3.214 \\
\hline $40 / 50$ & 0.4095 & 0.7893 & 0.9524 & 0.1768 & 0.50 & 000 & 0.82 & & 0.04 & 760 & 0.210 & & 0.5905 & & 3.214 \\
\hline $50 / 50$ & 0.4095 & 0.7893 & 0.9524 & 0.1768 & 0.50 & 000 & 0.82 & & 0.04 & & 0.210 & & 0.5905 & & 3.214 \\
\hline
\end{tabular}

guarantee convergence to at least three significant digits throughout, the numerical results reported in what follows were computed with fixed truncation orders $N_{\Psi}, N_{T}=40$.

\subsection{Verification}

In the second set of results, verification was pursued for the $x$ component of the velocity field, $u(x, y)$, at the cavity midplane along the vertical axis of symmetry $(x=0.5)$, and for the temperature field $T(x, y)$ at the cavity midplane along the horizontal axis of symmetry $(y=0.5)$. The GITT results are compared against those reported by Al-Najem et al. [10] and by Colaço et al. [14]. Figs. 2 and 3 show the velocity and temperature profiles for an external magnetic field, transversal to gravity acceleration $\left(\gamma=0^{\circ}\right)$, $\operatorname{Pr}=0.71$, for $\mathrm{Ha} \in\{0,10,15,25,50,100\}$ and for $\mathrm{Gr}=10^{4}$ and $\mathrm{Gr}=10^{6}$, respectively. Owing to different definitions adopted for the dimensionless velocity, the current GITT results for $u(x, y)$ are divided by $\mathrm{Gr}^{1 / 2}$ in order to compare with the results reported in Ref. [10]. ${ }^{2}$ From the analysis of Figs. 2 and 3 we may conclude that the current GITT results for both velocity and temperature profiles match to the graph scale the FVM and RBF results, respectively reported by Al-Najem et al. [10] and by Colaço et al. [14].

In the third set of results, we compare the GITT results obtained for the average Nusselt number at the hot vertical wall with those

\footnotetext{
2 Al-Najem et al. [10] defined the dimensionless velocities $U$ and $V$ as $U=u^{*} /\left(g \beta \Delta T^{*} L\right)^{1 / 2}$ and $V=v^{*} /\left(g \beta \Delta T^{*} L\right)^{1 / 2}$. Using our definitions for the dimensionless independent and dependent variables, it is easy to demonstrate that $u=u^{*} L / v$ and $v=v^{*} L / v$. After algebraic manipulations, one may demonstrate that $u=U\left(g \beta \Delta T^{*} L^{3} / v^{2}\right)^{1 / 2}=U G r^{1 / 2}$ and $v=V\left(g \beta \Delta T^{*} L^{3} / v^{2}\right)^{1 / 2}=V G r^{1 / 2}$.
}

reported in Refs. [10,14], for $\mathrm{Gr} \in\left\{10^{4}, 10^{6}\right\}, \operatorname{Pr}=0.71, \gamma=0^{\circ}$ and for different values of the Hartmann number, Ha. Table 4 reports the GITT results for $\overline{N u}_{\mathrm{x}=0}$ and the ones computed in Refs. [10,14]. For the lowest Grashof number, we verify that the current GITT results are in excellent agreement with those reported in Ref. [10]. The largest relative deviation is approximately $2.4 \%$ for $\mathrm{Ha}=25$. The GITT results are also in good agreement with the RBF results (with $15 \times 15$ centers) reported by Colaço et al. [14]; the largest relative deviation reaching $5.05 \%$ for $\mathrm{Ha}=50$. Larger deviations are noticed when comparing with the FVM results (with $41 \times 41$ control volumes) also reported by Colaço et al. [14], reaching $8 \%$ and $11.6 \%$, respectively, for $\mathrm{Ha}=10$ and $\mathrm{Ha}=25$. For the highest Grashof number, the GITT results for the average Nusselt number are lower than those reported in Ref. [10]. The relative deviation increases from $8.5 \%$ to $11.2 \%$ when the Hartmann number increases from 0 to 100 . Smaller deviations are verified when the GITT results are compared with the FVM results (with $41 \times 41$ control volumes) reported in Ref. [14], although the largest deviation reaches $24.7 \%$ for $\mathrm{Ha}=100$. When compared to the RBF results (with $25 \times 25$ centers) reported in Ref. [14], the largest deviation achieved is $14.2 \%$ for $\mathrm{Ha}=25$. In synthesis, larger deviations have been verified for the largest Grashof number and for larger Hartmann numbers, although larger differences are encountered for $\mathrm{Gr}=10^{4}$ when comparing the current GITT results with those obtained in Ref. [14] with FVM.

In the fourth set of results, the GITT results for the average Nusselt number $\overline{N u}_{\mathrm{x}=0}$ are compared against those of Pirmohammadi et al. [8], which reported in tabular form numerical results for the average Nusselt number at the hot vertical wall as a function of Rayleigh ( $\mathrm{Ra} \equiv \mathrm{GrPr}$ ) and Hartmann numbers, for a fixed value 

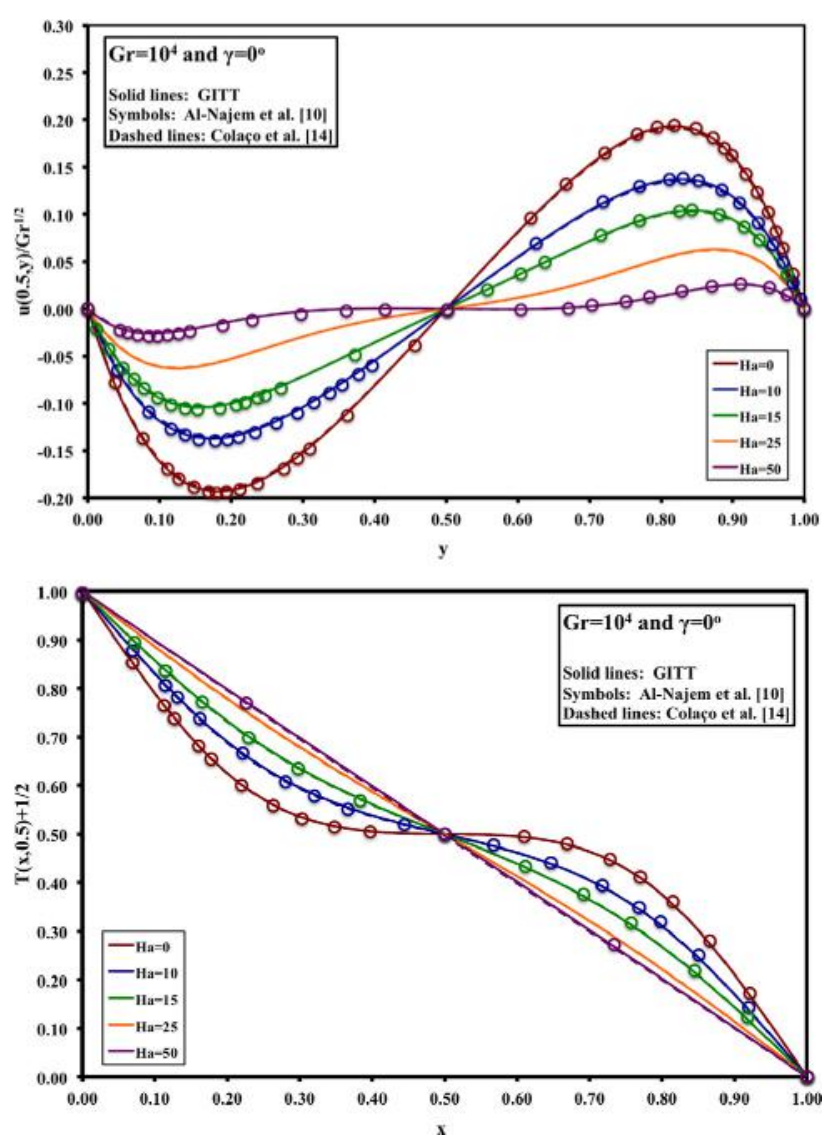

Fig. 2. Velocity and temperature distributions along cavity midplane vertical and horizontal axes of symmetry for $\mathrm{Gr}=10^{4}$ and $\gamma=0^{\circ}, u(0.5, y)$ and $T(x, 0.5)+1 / 2$. The present GITT results for the dimensionless velocity $u$ were divided by $\mathrm{Gr}^{1 / 2}$ to compare with those reported by Al-Najem et al. [10] and by Colaço et al. [14] (RBF with $15 \times 15$ centers).

of the Prandtl number $(\operatorname{Pr}=0.733)$. Those authors considered only the case of magnetic field transversal to gravity acceleration (i.e., $\gamma=0^{\circ}$ ). They solved the governing equations in the primitive variables formulation with the finite-volume method and the SIMPLER algorithm to treat the velocity-pressure coupling. The GITT results and those reported in Ref. [8] are summarized in Table 5. We may conclude that the GITT results for the average Nusselt number, although always smaller, are in good agreement with those reported by Pirmohammadi et al. [8]. The relative deviation generally increases as the Hartmann number increases. For Ra $=10^{4}$, the minimum and maximum relative deviations are, respectively, $-1.67 \%$ and $-2.08 \%$. When the Rayleigh number increases one order of magnitude $\left(\mathrm{Ra}=10^{5}\right)$, the relative deviation reaches $9.4 \%$ and $11.9 \%$ for the two larger Hartmann numbers. For the largest Rayleigh number, the relative deviation does not exceed $2.0 \%$ for the first three Hartmann numbers, notwithstanding it reaches $19.3 \%$ for $\mathrm{Ha}=300$.

In the final set of verification results, we compare the current GITT results with those reported in Han [13], which solved the MHD problem addressed here in a tilted cavity with an imposed magnetic field always parallel to gravity acceleration. The governing equations in the primitive variables formulation were solved with the finite-volume method with the SIMPLER algorithm. The particular case of a tilt angle equal to $90^{\circ}$ in Ref. [13] is equivalent to our problem with $\gamma$ set to $-90^{\circ}$ (see Fig. 1). ${ }^{3}$ For the particular

\footnotetext{
${ }^{3}$ It should be noticed that in Ref. [13] the cold and hot walls are at the boundaries $x=0$ and $x=1$, respectively. Hence, the main heat flow direction is from right to left, contrary to our case.
}
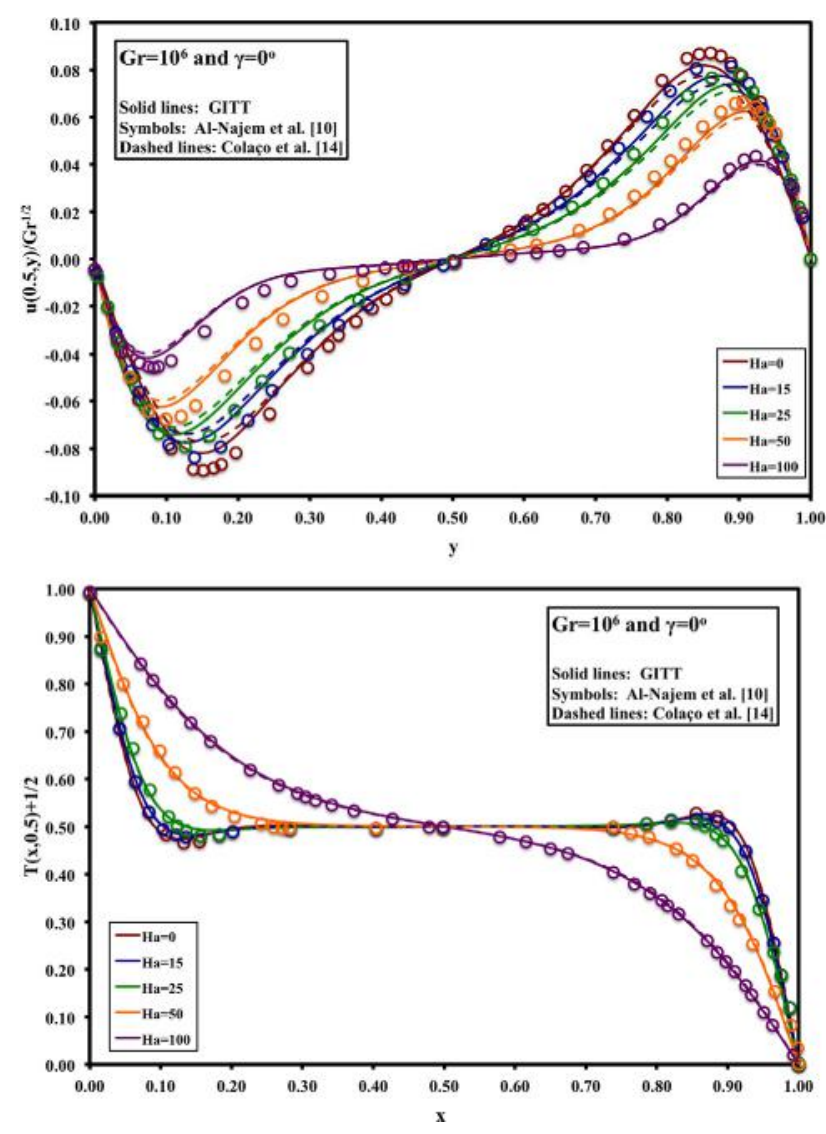

Fig. 3. Velocity and temperature distributions along cavity midplane vertical and horizontal axes of symmetry for $\mathrm{Gr}=10^{6}$ and $\gamma=0^{\circ}, u(0.5, y)$ and $T(x, 0.5)+1 / 2$. The present GITT results for the dimensionless velocity $u$ were divided by $\mathrm{Gr}^{1 / 2}$ to compare with those reported by Al-Najem et al. [10] and by Colaço et al. [14] (RBF with $25 \times 25$ centers)

case in which $\mathrm{Gr}=10^{6}, \mathrm{Ha}=0$ and $\mathrm{Pr}=0.733$, the computed GITT result for the average Nusselt number at the hot wall is 8.103 whereas the results reported in Ref. [13] are 8.13 and 8.10, respectively for a non-uniform $(51 \times 51)$ and uniform $(201 \times 201)$ meshes of control volumes. We may thus conclude that the current GITT results are also in good agreement with the results reported in the literature for the particular case in which the magnetic field is oriented parallel to gravity acceleration.

Fig. 4, which provides both verification and validation of the present simulation, shows the comparison for the dimensionless $y$-direction velocity component, $v(x, 0.5)$ along the cavity midplane $y=0.5 .{ }^{4}$ The solid line represents the GITT results, while the open circles represent the numerical predictions in Ref. [13] and the solid triangles denote the experimental data measured by Linthorst et al. [45] by means of laser doppler velocimetry. The numerical $y$ direction velocities shown in Fig. 4 are computed using the same dimensionless governing parameters estimated in the experiments performed by Linthorst et al. [45], namely, $\mathrm{Ra}=1.3 \times 10^{5}, \mathrm{Ha}=0$ and $\operatorname{Pr}=0.733$. One may conclude that the present GITT results agree quite well with both Han's numerical predictions and the experimental measurements reported in Ref. [45]. Notice that for the plot shown in Fig. 4, the hot wall is located at $x=1$. The GITT results

\footnotetext{
${ }^{4}$ Han [13] defined the dimensionless velocities $U$ and $V$ as $U=u^{*} / v_{0}{ }^{*}$ and $V=v^{*} / v_{0}{ }^{*}$ where the reference velocity $v_{0}^{*}$ is given by the ratio $\alpha / L$. Based on our definition of dimensionless velocities, algebraic manipulations yield the following relationships $u=U / \operatorname{Pr}$ and $v=V / \operatorname{Pr}$. Hence, the GITT results for the dimensionless velocity $v(x, 0.5)$ shown in Fig. 4 are multiplied by the Prandtl number to compare with Han's results [13].
} 
Table 4

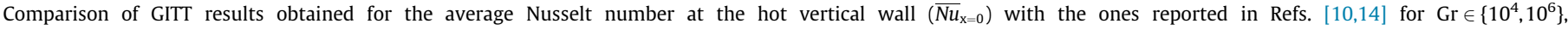
$\mathrm{Ha} \in\{0,10,25,50,100\}, \gamma=0^{\circ}$ and $\operatorname{Pr}=0.71$.

\begin{tabular}{|c|c|c|c|c|c|c|c|c|}
\hline \multirow[t]{2}{*}{ На } & \multicolumn{4}{|c|}{$\mathrm{Gr}=10^{4}$} & \multicolumn{4}{|c|}{$\mathrm{Gr}=10^{6}$} \\
\hline & GITT & Ref. [10] (FVM) & Ref. [14] (RBF) & Ref. [14] (FVM) & GITT & Ref. [10] (FVM) & Ref. [14] (RBF) & Ref. [14] (FVM) \\
\hline 0 & 2.011 & 2.01 & 2.02 & 2.06 & 8.016 & 8.76 & 9.21 & 7.98 \\
\hline 10 & 1.692 & 1.69 & 1.70 & 1.84 & 7.869 & 8.66 & 9.04 & 7.88 \\
\hline 25 & 1.167 & 1.14 & 1.17 & 1.32 & 7.139 & 8.04 & 8.32 & 7.39 \\
\hline 50 & 1.019 & 1.00 & 0.97 & 1.06 & 5.466 & - & - & - \\
\hline 100 & 1.002 & - & - & - & 3.214 & 3.76 & 3.54 & 4.27 \\
\hline
\end{tabular}

Table 5

Comparison of GITT results obtained for the average Nusselt number at the hot vertical wall $\left(\overline{N u}_{\mathrm{x}=0}\right)$ with the ones reported in Ref. [8] for $\mathrm{Ra} \in\left\{10^{4}, 10^{5}, 10^{6}\right\}$, $\mathrm{Ha} \in[0,300], \gamma=0^{\circ}$ and $\operatorname{Pr}=0.733$.

\begin{tabular}{lllll}
\hline $\mathrm{Ra}$ & Ha & GITT & Ref. [8] & Relative deviation (\%) \\
\hline $10^{4}$ & 0 & 2.246 & 2.29 & -1.92 \\
& 10 & 1.929 & 1.97 & -2.08 \\
& 50 & 1.038 & 1.06 & -2.08 \\
& 100 & 1.003 & 1.02 & -1.67 \\
$10^{5}$ & 0 & 4.530 & 4.62 & -1.95 \\
& 25 & 3.435 & 3.51 & -2.14 \\
& 100 & 1.241 & 1.37 & -9.42 \\
10 & 200 & 1.022 & 1.16 & -11.9 \\
& 0 & 8.841 & 8.9 & -0.66 \\
& 50 & 6.314 & 6.39 & -1.19 \\
& 150 & 2.587 & 2.64 & -2.01 \\
& 300 & 1.331 & 1.65 & -19.3 \\
\hline
\end{tabular}



Fig. 4. Comparison of dimensionless $y$-direction velocities $-v(x, 0.5) \operatorname{Pr}$ at the cavity midplane $y=0.5$ for $\mathrm{Ra}=1.3 \times 10^{5}, \mathrm{Ha}=0, \gamma=0^{\circ}$ and $\mathrm{Pr}=0.733$.

reproduce quite well the measured increasing-decreasing behavior of the $y$-direction velocity component near the hot wall.

\section{Conclusions}

A hybrid numerical-analytical solution has been obtained, based on eigenfunction expansions, for the coupled system of nonlinear partial differential equations governing the 2-D magnetohydrodynamic flow with heat transfer inside a square cavity, differentially heated at the sidewalls. The governing equations were rewritten in dimensionless form using the streamfunctiononly formulation. After integral transformation, the coupled system of nonlinear partial differential equations was reduced to a denumerable system of nonlinear fourth-order ordinary differential equations. Its numerical solution was obtained through robust and well-tested Fortran subroutines, with automatic relative error control, after truncating the series expansions for both streamfunction and temperature to a finite number of terms.

The convergence behavior of the hybrid solution for both the streamfunction and temperature fields at prescribed positions inside the cavity, as well as for the average Nusselt number at the hot wall, was carefully investigated in order to demonstrate the robustness of the proposed eigenfunction expansions. The GITT results reported here are fully converged to at least three significant digits in all cases considered. Four significant digits are fully converged for the average Nusselt number, which is the quantity of practical relevance in thermal engineering. Afterwards, a detailed verification and validation analysis was performed by comparing the current results obtained for fluid velocities and temperature, as well as for the average Nusselt number, against previous results (numerical and experimental) reported in the literature. Quite good agreement was achieved in most situations, with the GITT results for the average Nusselt number in general lower than those computed by other authors with FVM and RBF schemes. Relative deviations in general increased for higher Grashof and Hartmann numbers.

The proposed eigenfunction expansion approach may be extended to more involved magneto-hydrodynamic natural convection problems to investigate, in future works, the effects of various parameters on heat transfer rate, including cavity inclination angle and aspect ratio, internal volumetric heat generation rate, spatial variation of temperature and/or heat flux at the boundaries, spatial variation of thermophysical properties, and irregular geometries.

\section{Acknowledgements}

The authors (João N. N. Quaresma and Renato M. Cotta) would like to acknowledge the financial support provided by the Brazilian sponsoring agencies CNPq and FAPERJ.

Carlos Frederico T. Matt would like to acknowledge the Electric Power Research Center (CEPEL) for the financial support of the research described in the current manuscript. He would also like to acknowledge Prof. L. F. Shampine from the Mathematics Department at Southern Methodist University, Dallas, TX, and Profs. P. H. Muir and H. Xu from Mathematics and Computing Science at Saint Mary's University, Halifax, Nova Scotia, Canada, for freely licensing the Fortran 90 subroutine BVP SOLVER to solve two-point boundary-value problems.

\section{References}

[1] P.A. Davidson, An Introduction to Magnetohydrodynamics, Cambridge Texts in Applied Mathematics, Cambridge University Press, Cambridge, UK, 2001.

[2] G.S. Dulikravich, S.R. Lynn, Unified electro-magneto-fluid dynamics (EMFD): introductory concepts, Int. J. Non-Linear Mech. 32 (1997) 913-922.

[3] G.S. Dulikravich, S.R. Lynn, Unified electro-magneto-fluid dynamics (EMFD): a survey of mathematical models, Int. J. Non-Linear Mech. 32 (1997) 923-932.

[4] A.C. Eringen, G.A. Maugin, Electrodynamics of Continua I: Foundations and Solid Media, Springer, New York, 1990.

[5] A.C. Eringen, G.A. Maugin, Electrodynamics of Continua II: Fluids and Complex Media, Springer, New York, 1990. 
[6] G.C. Bourantas, E.D. Skouras, V.C. Loukopoulos, G.C. Nikiforidis, An accurate, stable and efficient domain-type meshless method for the solution of MHD flow problems, J. Comput. Phys. 228 (2009) 8135-8160.

[7] M. Pirmohammadi, M. Ghassemi, Effect of magnetic field on convection heat transfer inside a tilted square enclosure, Int. Commun. Heat Mass Transfer 36 (2009) 776-780.

[8] M. Pirmohammadi, M. Ghassemi, G.A. Sheikhzadeh, The effect of a magnetic field on buoyancy-driven convection in differentially heated square cavity, in: Proceedings of the 14th Symposium on Electromagnetic Launch Technology, June 10-13, Victoria, BC, 2008.

[9] S. Alchaar, P. Vasseur, E. Bilgen, The effect of a magnetic field on natural convection in a shallow cavity heated from below, Chem. Eng. Commun. 134 (1995) 195-209.

[10] N.M. Al-Najem, K.M. Khanafer, M.M. El-Refaee, Numerical study of laminar natural convection in tilted enclosure with transverse magnetic field, Int. J. Numer. Meth. Heat Fluid Flow 8 (1998) 651-672.

[11] H. Ozoe, K. Okada, The effect of the direction of the external magnetic field on the three-dimensional natural convection in a cubical enclosure, Int. J. Heat Mass Transf. 32 (1989) 1939-1954.

[12] G.M. Oreper, J. Szekely, The effect of an externally imposed magnetic field on buoyancy driven flow in a rectangular cavity, J. Cryst. Growth 64 (1983) 505515.

[13] C.Y. Han, Effect of a magnetic field on natural convection of an electrically conducting fluid in a tilted cavity, J. Korean Phys. Soc. 55 (2009) 2193-2199.

[14] M.J. Colaço, G.S. Dulikravich, H.R.B. Orlande, Magnetohydrodynamic simulations using radial basis functions, Int. J. Heat Mass Transf. 52 (2009) 5932-5939.

[15] E.J. Kansa, Multiquadrics - a scattered data approximation scheme with applications to computational fluid dynamics - II solutions to parabolic, hyperbolic and elliptic partial differential equations, Comput. Math. Appl. 19 (1990) 149-161.

[16] R.M. Cotta, Hybrid numerical-analytical approach to nonlinear diffusion problems, Numer. Heat Transfer, Part B 127 (1990) 217-226.

[17] J.S. Pérez Guerrero, R.M. Cotta, Integral transform method for Navier-Stokes equations in stream function-only formulation, Int. J. Numer. Meth. Fluids 15 (1992) 399-409.

[18] R.M. Cotta, Integral Transforms in Computational Heat and Fluid Flow, CRC Press, Boca Raton, Florida, 1993.

[19] R.M. Cotta, Benchmark results in computational heat and fluid flow - the integral transform method, Int. J. Heat Mass Transfer (Invited Paper) 37 (1994) 381-394.

[20] R.M. Cotta, M.D. Mikhailov, Heat Conduction: Lumped Analysis, Integral Transforms, Symbolic Computation, Wiley-Interscience, Chichester, UK, 1997.

[21] R.M. Cotta, The Integral Transform Method in Thermal and Fluid Sciences and Engineering, Begell House, New York, 1998.

[22] C.A.C. Santos, J.N.N. Quaresma, J.A. Lima, Benchmark Results for Convective Heat Transfer in Ducts: The Integral Transform Approach, E-papers, Rio de Janeiro, 2001.

[23] R.M. Cotta, M.D. Mikhailov, Hybrid methods and symbolic computations, in: W.J. Minkowycz, E.M. Sparrow, J.Y. Murthy (Eds.), Handbook of Numerical Heat Transfer, second ed., John Wiley, New York, 2006, Chapter 16.

[24] R.M. Cotta, D.C. Knupp, C.P. Naveira-Cotta, Analytical Heat and Fluid Flow in Microchannels and Microsystems, Mechanical Engineering Series, Springer, New York, 2016.
[25] C. Baohua, R.M. Cotta, Integral transform analysis of natural convection in porous enclosures, Int. J. Num. Meth. Fluids 17 (1993) 787-801.

[26] L.S.B. Alves, R.M. Cotta, Transient natural convection inside porous cavities:hybrid numerical-analytical solution and mixed symbolic-numerical computation, Num. Heat Transfer, Part A - Appl. 38 (1) (2000) 89-110.

[27] H. Luz Neto, J.N.N. Quaresma, R.M. Cotta, Natural convection in threedimensional porous cavities: integral transform method, Int. J. Heat Mass Transfer 45 (14) (2002) 3013-3032.

[28] M.A. Leal, J.S. Pérez Guerrero, R.M. Cotta, Natural convection inside twodimensional cavities: The integral transform method, Commun. Numer. Methods Eng. 15 (1999) 113-125.

[29] M.A. Leal, H.A. Machado, R.M. Cotta, Integral transform solutions of transient natural convection in enclosures with variable fluid properties, Int. J. Heat Mass Transf. 43 (2000) 3977-3990.

[30] C. An, C.B. Vieira, J. Su, Integral transform solution of natural convection in a square cavity with volumetric heat generation, Braz. J. Chem. Eng. 30 (2013) 883-896.

[31] J.A. Lima, M.G.O. Rêgo, On the integral transform solution of low-magnetic MHD flow and heat transfer in the entrance region of a channel, Int. J. NonLinear Mech. 50 (2013) 25-39.

[32] C. An, J. Su, Dynamic response of clamped axially moving beams: integral transform solution, Appl. Math. Comput. 218 (2011) 249-259.

[33] C. An, J. Su, Dynamic response of axially moving Timoshenko beams: integral transform solution, Appl. Math. Mech. 35 (2014) 1421-1436.

[34] C. An, J. Su, Dynamic analysis of axially moving orthotropic plates: integral transform solution, Appl. Math. Comput. 228 (2014) 489-507.

[35] C.F. Matt, Combined classical and generalized integral transform approaches for the analysis of the dynamic behavior of a damaged structure, Appl. Math. Model. 37 (2013) 8431-8450.

[36] C.F. Matt, Simulation of the transverse vibrations of a cantilever beam with an eccentric tip mass in the axial direction using integral transforms, Appl. Math. Model. 37 (2013) 9338-9354.

[37] C.F. Matt, Transient response of general one-dimensional distributed systems through eigenfunction expansion with an implicit filter scheme, Appl. Math. Model. 39 (2015) 2470-2488.

[38] J.J. Gu, C. An, M.L. Duan, C. Levi, J. Su, Integral transform solutions of dynamic response of a clamped-clamped pipe conveying fluid, Nucl. Eng. Des. 254 (2013) 237-245

[39] C.F. Matt, On the application of generalized integral transform technique to wind-induced vibrations on overhead conductors, Int. J. Numer. Meth. Eng. 78 (2009) 901-930.

[40] M.D. Mikhailov, M.N. Özisik, Unified Analysis and Solutions of Heat and Mass Diffusion, John Wiley, New York, 1984.

[41] J.J.G. Silva, E.N. Macêdo, J.N.N. Quaresma, R.M. Cotta, Hybrid solutions for MHD natural convection in cavities, in: Proceedings of the 21st Brazilian Congress of Mechanical Engineering - COBEM2011, Natal, 2011.

[42] S. Wolfram, Mathematica v. 10, Wolfram Research Inc., 2016.

[43] IMSL $^{\circledR}$ Fortran Numerical Library, Version 7.1.0, Rogue Wave Software Inc. Boulder, USA, 2014

[44] L.F. Shampine, P.H. Muir, H. Xu, A user-friendly Fortran BVP solver, J. Numer. Anal., Ind. Appl. Math. 1 (2006) 201-217.

[45] S.J.M. Linthorst, W.M.M. Schinkel, C.J. Hoogendoorn, Flow structure with natural convection in inclined air-filled enclosures, J. Heat Transfer 103 (1981) 535-539. 